ARTICLE

DOI: $10.1038 /$ s41467-017-02164-1

\title{
Poln O-GlcNAcylation governs genome integrity during translesion DNA synthesis
}

\author{
Xiaolu Ma', Hongmei Liu ${ }^{2}$, Jing Li id ${ }^{3}$, Yihao Wang ${ }^{4}$, Yue-He Ding ${ }^{5}$, Hongyan Shen (1) ${ }^{1}$, Yeran Yang ${ }^{1}$,

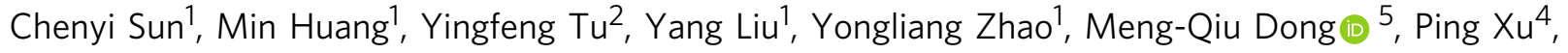 \\ Tie-Shan Tang ${ }^{2} \&$ Caixia Guo ${ }^{1}$
}

DNA polymerase $\eta$ (Pol $\eta$ ) facilitates translesion DNA synthesis (TLS) across ultraviolet (UV) irradiation- and cisplatin-induced DNA lesions implicated in skin carcinogenesis and chemoresistant phenotype formation, respectively. However, whether post-translational modifications of Pol $\eta$ are involved in these processes remains largely unknown. Here, we reported that human Poln undergoes O-GIcNAcylation at threonine 457 by O-GlcNAc transferase upon DNA damage. Abrogation of this modification results in a reduced level of CRL4CDT2. dependent Poln polyubiquitination at lysine 462, a delayed p97-dependent removal of Poln from replication forks, and significantly enhanced UV-induced mutagenesis even though Pol $\eta$ focus formation and its efficacy to bypass across cyclobutane pyrimidine dimers after UV irradiation are not affected. Furthermore, the O-GIcNAc-deficient T457A mutation impairs TLS to bypass across cisplatin-induced lesions, causing increased cellular sensitivity to cisplatin. Our findings demonstrate a novel role of Poln O-GlcNAcylation in TLS regulation and genome stability maintenance and establish a new rationale to improve chemotherapeutic treatment.

\footnotetext{
${ }^{1}$ CAS Key Laboratory of Genomics and Precision Medicine, Beijing Institute of Genomics, University of Chinese Academy of Sciences, Chinese Academy of Sciences, Beijing 100101, China. ${ }^{2}$ State Key Laboratory of Membrane Biology, Institute of Zoology, University of Chinese Academy of Sciences, Chinese Academy of Sciences, Beijing 100101, China. ${ }^{3}$ Beijing Key Laboratory of DNA Damage Response, College of Life Sciences, Capital Normal University, Beijing 100048, China. ${ }^{4}$ State Key Laboratory of Proteomics National Center for Protein Sciences Beijing, Beijing Proteome Research Center, National Engineering Research Center for Protein Drugs, Beijing Institute of Radiation Medicine, Beijing 102206, China. ${ }^{5}$ National Institute of Biological Sciences (Beijing), Beijing 102206, China. Xiaolu Ma, Hongmei Liu, and Jing Li contributed equally to this work. Correspondence and requests for materials should be addressed to T.-S.T. (email: tangtsh@ioz.ac.cn) or to C.G. (email: guocx@big.ac.cn)
} 
T ranslesion DNA synthesis (TLS) is one mode of DNA damage tolerance, which utilizes multiple specialized DNA polymerases to replicate damaged DNA to maintain genome integrity. One such polymerase, polymerase $\eta$ (Pol $\eta$ ), is specifically required for the accurate replicative bypass of cyclobutane pyrimidine dimers (CPDs) in DNA generated by ultraviolet (UV) radiation ${ }^{1-4}$. Mammalian Poln possesses a polymerase catalytic domain in its $\mathrm{N}$-terminus ${ }^{5,6}$, a proliferating cell nuclear antigen (PCNA)-interacting region and a ubiquitinbinding zinc finger domain (UBZ) responsible for its interaction with monoubiquitinated PCNA (mUb-PCNA) in its Cterminus ${ }^{7,8}$. Compelling evidence has shown that Poln is recruited to stalled replication forks after UV ${ }^{7,9-11}$ and cisplatin (cisdiamminedichloroplatinum, CDDP) exposure ${ }^{12,13}$. DNA damage-induced Poln focus formation is dependent upon its UBZ domains $^{7}$ and the RAD18 protein ${ }^{14}$. The biological significance of Poln in the bypass of UV-induced CPDs is manifested by diseases in mice and humans lacking normal Pol $\eta$ protein ${ }^{15-17}$. Additionally, Poln is capable of replicating across other types of DNA damage in vitro, including CDDP-induced GpG adducts $(\mathrm{Pt}-\mathrm{GG})^{6,18,19}$. Consistently, the expression level of Poln is inversely correlated with CDDP treatment efficacy ${ }^{20,21}$. However, since Poln replicates undamaged DNA with a high error rate of $10^{-2}-10^{-3}$, its recruitment and residence at replication forks has to be stringently regulated ${ }^{6}$. So far, it is known that, in addition to protein-protein interactions $s^{4,22-24}$, protein post-translational modifications (PTMs), such as phosphorylation ${ }^{13,25}$ and ubiquitination $^{8,26,27}$, fine-tune Poln recruitment and bypass of CPD lesions after UV radiation. Given its reduced affinity for the DNA beyond the CPD, Poln dissociation after TLS has been suggested to be its intrinsic property ${ }^{28}$. Moreover, deubiquitination of mUb-PCNA by USP $1^{29}$, USP $10^{30}$, or interferon-stimulated gene 15 modification of PCNA has also been suggested to dictate TLS termination ${ }^{30}$. Nevertheless, given that UV-induced mUb-PCNA can persist long after TLS is completed ${ }^{31}$, it remains a conundrum how disassembly of Poln happens in the persistence of mUbPCNA.

Recently, O-linked $\beta$-N-acetylglucosamine (O-GlcNAc) to serine and threonine residues of proteins, termed O-GlcNAcylation ${ }^{32}$, is emerging as a key regulator of diverse cellular processes, such as signal transduction and proteasomal degradation ${ }^{33-35}$. Analogous to phosphorylation, O-GlcNAcylation is highly dynamic and has extensive crosstalk with other PTM forms ${ }^{32}$. O-GlcNAc cycling is modified by only one O-GlcNAc transferase (OGT) and only one O-GlcNAcase (OGA) in mammals. Its donor substrate, UDPGlcNAc, exists in high intracellular concentrations. Aberrant OGlcNAcylation has been linked to a plethora of human diseases, including cancer ${ }^{36,37}$. Recently, OGT has been found to be recruited to sites of DNA damage ${ }^{38}$ and several proteins involved in DNA damage response (DDR) have been reported to be OGlcNAcylated ${ }^{35,38-40}$. O-GlcNAcylation of H2AX is further found to interfere with its phosphorylation ${ }^{38}$. However, much remains a mystery as for the role of O-GlcNAcylation in the DDR.

In this study, we found that OGT interacts with Poln and promotes Poln O-GlcNAcylation at T457. Although T457A mutation does not impair Poln bypass across CPD lesions, it unexpectedly restrains p97-dependent Poln removal from replication forks after TLS is completed, leading to an increased mutation frequency after UV irradiation. Poln also interacts with DDB1 and CDT2. Intriguingly, T457A mutation significantly attenuates Lys48 (K48)-linked Poln polyubiquitination catalyzed by Cullin 4-RING Ligase (CRL4)-DDB1-CDT2 (CRL4 ${ }^{\mathrm{CDT} 2}$ ), explaining the prolonged retention of the Poln T457A mutant at UV-damaged chromatin. Through quantitative mass spectrometry (MS), we found that the Poln T457A mutant displays an obvious reduction in ubiquitination at K462, revealing a novel crosstalk between Poln O-GlcNAcylation and ubiquitination, which modulates Poln disassembly from replication forks. Additionally, T457A mutation also causes a TLS deficiency upon CDDP exposure, accompanied with a reduced DNA replication rate. Therefore, O-GlcNAcylation plays an unexpected role in TLS polymerase switching, adding a further layer of regulation that elaborately controls TLS and genome stability in vivo.

\section{Results}

OGT binds to Poln and promotes Poly O-GlcNAcylation at T457. To identify novel proteins that may regulate Poln functions in vivo, we transfected HEK293T cells with a 2xFlag-Poln expression vector and performed immunopurification using the nuclear extracts ${ }^{41}$. Affinity-purified proteins associated with FlagPoln were separated by sodium dodecyl sulfate-polyacrylamide gel electrophoresis (SDS-PAGE) and revealed by silver staining (Fig. 1a). Two indicated regions enriched with bands not observed in the affinity-purified control extracts were cut and several proteins including OGT were identified via Liquid chromatography-tandem MS (LC-MS/MS) analysis. We then performed co-immunoprecipitation (Co-IP) assay to validate OGT/Poln interaction. Endogenous OGT was found to bind to Flag-Poln but not Flag tag alone, although the immunoprecipitated OGT only represents a small fraction $(<1 \%)$ of that in cells (Fig. 1b). OGT has been shown to relocate to the sites of DNA damage after ionizing radiation treatment. We then checked whether OGT is involved in UV-induced DDR. As shown in Fig. 1c, we found that the chromatin binding of OGT was enhanced after UV treatment. Interestingly, knockdown of OGT did not affect UV-induced Pol $\eta$ focus formation at $8 \mathrm{~h}$ but causing a significantly higher extent of Poln foci at $24 \mathrm{~h}$ post-UV compared with negative control (siNC) (Fig. 1d), suggesting that OGT might regulate Poln function after UV irradiation.

Given that OGT can add O-GlcNAc to target proteins, exogenously expressed Flag-Pol $\eta$ in 293-T cells was immunoprecipitated under a denaturing condition to assess its $\mathrm{O}$ GlcNAcylated level through immunoblotting using anti-OGlcNAc antibody. A band corresponding to O-GlcNAcylated Poln was observed (Fig. 1e). After treating the cells with glucose and Thiamet-G, the OGA inhibitor that suppresses the reversible removal of O-GlcNAc moiety from proteins, we found that Poln O-GlcNAcylation was substantially enriched (Fig. 1e). Additionally, 293T cells transfected with green fluorescent protein (GFP)Poln were cultured in media supplemented with different concentrations of glucose followed by IP under an undenaturing condition. We found that Poln O-GlcNAcylation level positively correlates with the glucose concentration in the medium (Fig. 1f), consistence with the fact that glucose is an important source of UDP-GlcNAc, the high-energy donor substrate of OGT. To exclude the possibility that Poln O-GlcNAcylation might be caused by its overexpression, we took advantage of an inducible XP30RO-Poln cell line ${ }^{42}$ to express SFB (streptavidin-Flag-S protein)-tagged Poln close to the endogenous level through supplementing Doxycycline $\left(0.01 \mu \mathrm{g} \mathrm{ml}^{-1}\right)$ in the medium. The result indicated that Pol $\eta$ when expressed at the endogenous level was also O-GlcNAcylated (Supplementary Fig. 1a).

To identify the potential O-GlcNAcylated residue(s) in Poln, 293T cells were transfected with Flag-Poln, and O-GlcNAcylation was enriched by treating the cells with Thiamet-G and glucose ${ }^{43}$. The immunoprecipitated Flag-Pol $\eta$ were divided into two groups: one was separated on SDS-PAGE and the expected Poln band was cut out for in-gel digestion and higher-energy collision dissociation tandem MS (HCD-MS) analysis, the other one was glycine eluted and subjected to HCD-MS analysis directly (Supplementary Fig. 1b, c). Results from both groups (with $92.8 \%$ or $86.4 \%$ Poln protein 
a

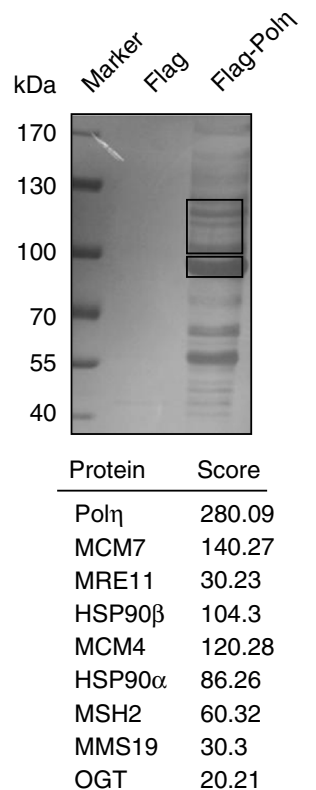

b
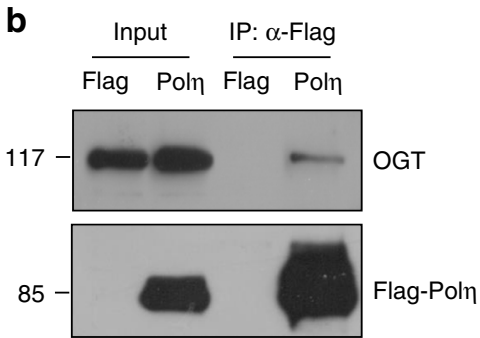

d

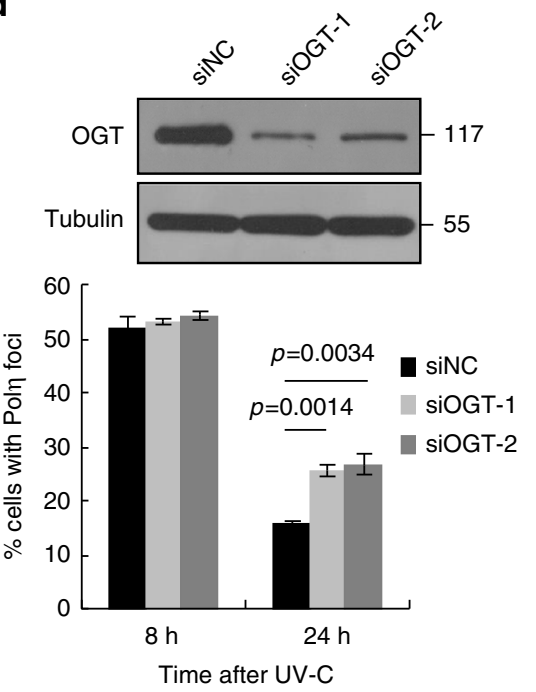

e

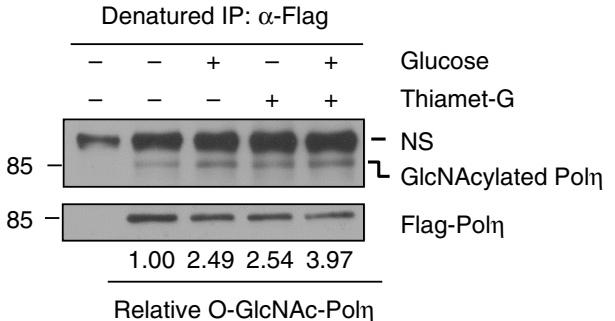

f

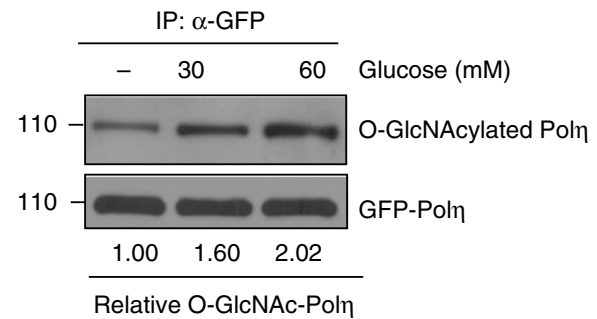

g

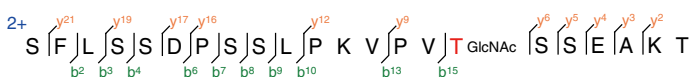

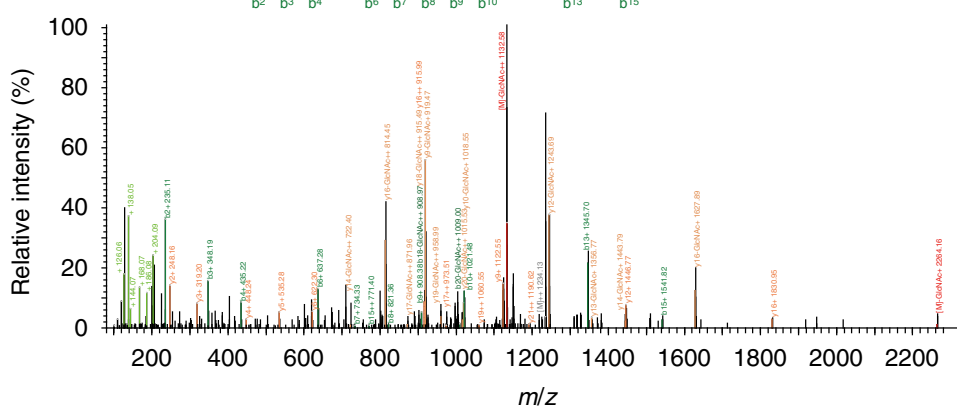

h

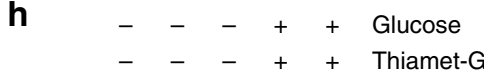

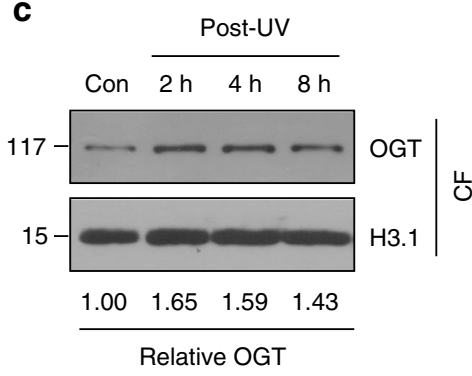
GFP WT 5A WT 5A

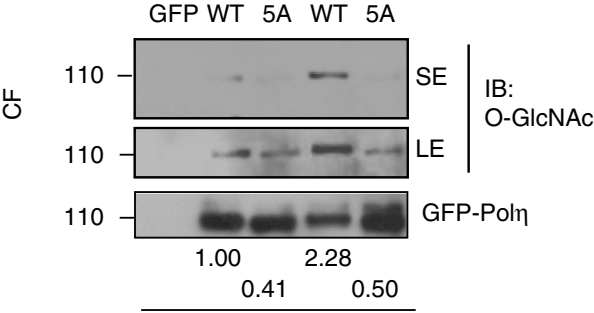

Relative O-GIcNAc-Poln i

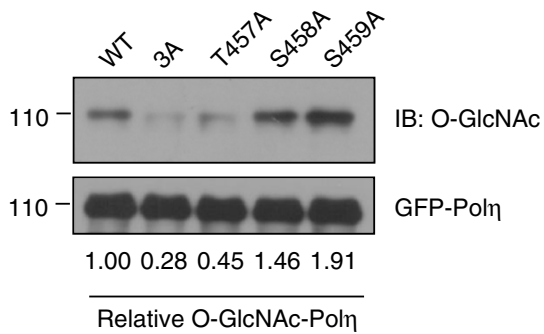

Fig. 1 Poln interacts with OGT and is subject to O-GIcNAcylation predominantly at T457. a The nuclear extracts of 293T cells expressing Flag-Poln were immunoprecipitated. The indicated bands (black rectangles) were cut and analyzed via mass spectrometric analysis. b $293 \mathrm{~T}$ cell lysates expressing Flag-Poln were immunoprecipitated and detected with anti-OGT and anti-Flag antibodies. The input included $2 \%$ of the cell lysate used. c U2OS cells were irradiated with UVC $\left(15 \mathrm{~J} \mathrm{~m}^{-2}\right)$ and harvested at different time points later. The chromatin fractions were extracted followed by immunoblotting with OGT and H3.1 antibodies. d U2OS cells were transfected with siOGT or siNC oligos. The OGT protein level was detected by western blot. The knockdown cells were transfected with GFP-Poln, irradiated with UVC $\left(15 \mathrm{~J} \mathrm{~m}^{-2}\right)$ and further incubated for $24 \mathrm{~h}$. The proportions of GFP-Poln-expressing cells with $>30$ foci were determined. Data represent means \pm SEM from three independent experiments. e $293 T$ cells transfected with Flag-Pol $\eta$ or empty vector were incubated with Thiamet-G and glucose. The cell lysates were denatured and immunoprecipitated with anti-Flag M2 beads followed by immunoblotting with O-GlcNAc and Flag antibodies. NS: non-specific band. f 293T cells transfected with GFP-Pol $\eta$ were treated with Thiamet-G and different concentrations of glucose. The cell lysates were immunoprecipitated with GFP-Trap A followed by immunoblotting with O-GlcNAc and GFP antibodies. g 293T cells transfected with Flag-Poln or empty vector were treated with Thiamet-G and glucose. The cell lysates were immunoprecipitated with anti-Flag agarose followed by HCD-MS analysis. The tryptic peptide containing a HexNAc (+203.08 Da) was detected. The band y-type product ions were marked on the spectrum and also illustrated along the peptide sequence shown on top of the spectrum. $\mathbf{h}$, $\mathbf{i} 293 \mathrm{~T}$ cells transfected with the indicated GFP-Poln constructs were treated as in $\mathbf{g}$. The cell lysates were immunoprecipitated with GFP-Trap A followed by immunoblotting with O-GIcNAc and GFP antibodies. SE short exposure, LE long exposure 
sequence coverage, respectively) revealed that Poln OGlcNAcylation mainly occurs on a peptide (SFLSSDPSSLPKVPVTSSEAKTQGSGPAVT) spanning residues 442-471 (Fig. 1g). To further determine the potential modification site(s), we generated a mutated Pol $\eta$ construct (Poln-5A), in which three serine $(S)$ and two threonine $(T)$ residues within the O-GlcNAc-modified peptide were mutated to alanines (A) (T457A/S458A/S459A/S466A/T471A). Wild-type (WT) and Pol $\eta-5 \mathrm{~A}$ constructs were then transfected into $293 \mathrm{~T}$ cells and their O-GlcNAcylation levels were compared. As shown in Fig. 1h, the O-GlcNAcylation level of Poln-5A was significantly reduced compared to that of WT in the absence or presence of Thiamet-G and glucose, supporting the notion that the majority of Poln O-GlcNAcylation occur within these residues.

Interestingly, both of $\mathrm{S} 466 \mathrm{~A}$ and $\mathrm{T} 471 \mathrm{~A}$ single mutants exhibited an increased Poln O-GlcNAcylation, hinting that these two residues were not the major sites for this modification (Supplementary Fig. 1d). We then generated another mutated Poln construct (Poln-3A) (T457A/S458A/S459A) and found that Poln-3A also manifested a markedly reduced level of $\mathrm{O}$ GlcNAcylation as Pol $\eta-5 A$ (Supplementary Fig. 1e). Furthermore, we generated three single mutants (T457A, S458A, and S459A) and assessed their O-GlcNAcylation levels. Intriguingly, T457A mutant, but not S458A or S459A mutant, showed a dramatically diminished level of Poln O-GlcNAcylation (Fig. 1i). Thus it is highly likely that T457 is the major O-GlcNAcylation site of Poln. T457 is located at the PVT/S (proline-valine-threonine/serine) motif that has been reported to be conserved in about half of the O-GlcNAcylated proteins identified to date ${ }^{32}$. Additionally, we found that T457A, 3A, and 5A mutants manifest reduced associations with OGT (Supplementary Fig. 1f). Furthermore, we mapped the regions within Poln responsible for its interaction with OGT by using Flag-tagged WT Pol $\eta$ and a series of Pol $\eta$ deletion mutants (Supplementary Fig. 2a). Co-IP experiments revealed that the $\mathrm{N}$-terminal fragment spanning the whole catalytic domain of Pol $\eta$ is required for its interaction with OGT (Supplementary Fig. 2b). Similarly, we also mapped the regions within OGT required for its association with Poln by using Myc-tagged WT OGT and a series of OGT deletion mutants (Supplementary Fig. 2c). Co-IP assays showed that the first two TPR regions of OGT are required for its association with Poln (Supplementary Fig. 2d).

T457A has no effect on Poln bypass CPDs. To examine whether O-GlcNAc modification of Poln participates in DDR, 293T cells expressing GFP-Poln were treated with UVC followed by an IP with anti-GFP antibody. We found that O-GlcNAcylation of Poln was dramatically increased after UV irradiation (Fig. 2a, top panel), which also exhibited a dynamic change after UV (Fig. 2a, bottom panel). Moreover, interaction between Poln and OGT was substantially elevated in $293 \mathrm{~T}$ cells transfected with Flag-Poln under UV radiation (Fig. 2b). These data suggest that O-GlcNAc modification of Poln plays a potential role in cellular response to UV exposure. To investigate the functional importance of Poln O-GlcNAcylation upon UV irradiation, we transfected WT and T457A-mutated GFP-Pol $\eta$ into U2OS cells and compared their focus formation after UV treatment. No significant difference in their focus formation between WT and T457A Pol $\eta$ was detected at $8 \mathrm{~h}$ after UV irradiation (Fig. 2c), suggesting that abolishment of Poln O-GlcNAcylation by T457A does not affect UV-induced

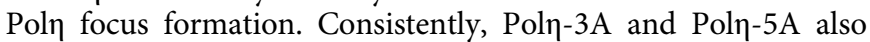
failed to show any obvious changes in focus formation post-UV irradiation relative to WT Poln (Fig. 2c).

To determine whether T457A mutation affects the ability of Pol $\eta$ to bypass CPD lesions, we established WT or T457A GFP-

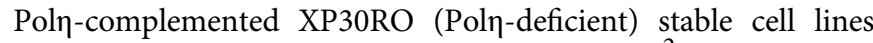
(Fig. 2d) and treated the cells with UVC $\left(5 \mathrm{~J} \mathrm{~m}^{-2}\right)$. As shown in Fig. 2e, although unreplicated CPDs persisted in the GFPexpressing XP30RO cells, they were undetectable in the XP30RO cells expressing either WT or T457A Poln at $4 \mathrm{~h}$ post-UV. It has been reported that TLS is involved in the disappearance of RPA foci ${ }^{4}$, which reflects the ssDNA regions in the chromatin. RPA signals were further examined in the above treated cells. Compared to GFP-expressing control cells that had a relative higher percentage of RPA foci-positive cells at either 4 or $24 \mathrm{~h}$ post-UV irradiation, WT or T457A Poln-expressing cells only manifested scanty RPA signals at those time points (Fig. 2f, g). These data suggested that T457A mutation does not compromise Poln ability to bypass CPD lesions.

T457A impairs Poln removal from chromatin after UV. Although loss of Poln O-GlcNAcylation caused by T457A does not impair Pol $\eta$ focus formation at $8 \mathrm{~h}$ after UV irradiation, we

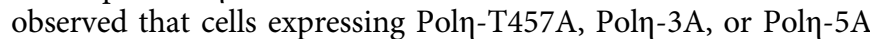
displayed a significantly higher extent of Poln foci at $24 \mathrm{~h}$ postUV compared with that of WT control (Fig. 3a). In line with this, our chromatin fractionation result revealed that T457A mutation remarkably impaired the removal of Poln from chromatin at $24 \mathrm{~h}$ post-UV (Supplementary Fig. 3a). FACS analysis showed that the cells expressing WT or T457A Pol $\eta$ had comparable cell cycle profiles at $24 \mathrm{~h}$ post-UV (Supplementary Fig. $3 \mathrm{~b}$ ), excluding the possibility that the increased Poln foci at that time is the result of a higher proportion of cells in S phase. O-GlcNAcylation often competes with phosphorylation in many biological processes, and to exclude the possibility that the phenotype may result from the loss of phosphorylation at T457, we constructed two phosphomimetic Poln mutants by replacing Thr457 with Asp (T457D) or Glu (T457E). We found that analogous to T457A mutant, T457D and T457E Poln still displayed a significantly higher extent of Pol $\eta$ foci at $24 \mathrm{~h}$ after UV (Supplementary Fig. 3c). We then speculated that the higher extent of Poln foci at $24 \mathrm{~h}$ post-UV might be due to their aberrant disassociation from replication forks after TLS is completed. Recently, several studies have reported that Poln disassociation from chromatin is modulated by an AAA-ATPase p97-UFD1-NPL4 complex, which acts as a K48 ubiquitin-chain-dependent protein segregase to remove polyubiquitinated DNA-binding proteins at the sites of DNA damage $^{45-47}$. We found that 5A, T457A, and WT Poln manifested similar associations with either UFD1 (Fig. 3b and Supplementary Fig. 4a) or NPL4 (Fig. 3c and Supplementary Fig. 4b), the two core adaptor factors for p97 to process its K48polyubiquitinated substrates ${ }^{47}$. Interestingly, the polyubiquitination level of Poln was significantly reduced when WT Poln was mutated to T457A or 3A or 5A (Fig. 3d). Using ubiquitin chainspecific K48 antibody, we further confirmed that ablation of Pol $\eta$ O-GlcNAcylation at T457 significantly decreased its K48polyubiquitination (Fig. 3d), which might restrain the removal of Poln from replication forks after TLS is completed.

Since Pol $\eta$ replicates undamaged DNA with very low fidelity ${ }^{6,48}$, we speculated that failure of Poln disassembly from replication forks in time might cause excessive TLS and increased mutation frequencies. To test that, a mutagenesis assay based on a UV-irradiated shuttle vector pSP189 that carries a mutant supFsuppressor tRNA33 was performed ${ }^{49}$. We found that expressing WT but not T457A Pol $\eta$ in XP30RO cells caused a significant reduction in the mutation frequency after exposure to UVC (400 $\mathrm{J} \mathrm{m}^{-2}$ ) (Fig. 3e). We also analyzed whether T457A mutation would affect cellular resistance to UV irradiation. Unlike WT Poln that can completely rescue the UV hypersensitivity in XP30RO cells, T457A mutant only displayed a partial rescue 
a

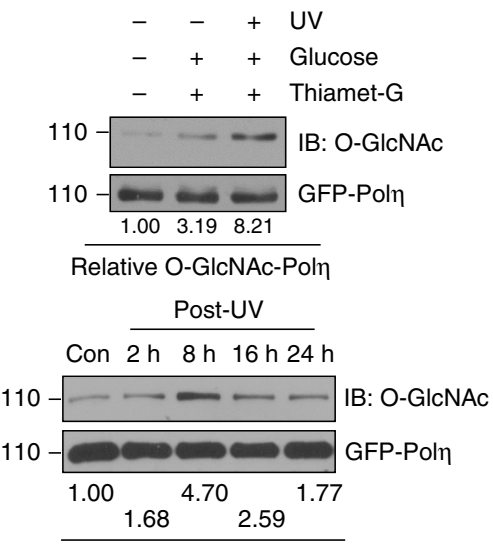

Relative O-GlcNAc-Poln
C

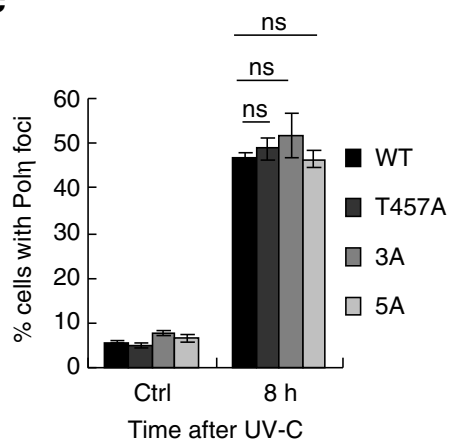

Time after UV-C d

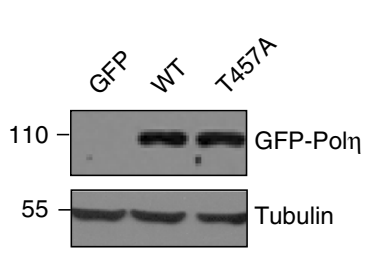

e

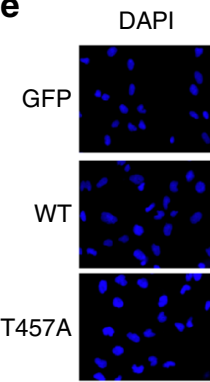

b

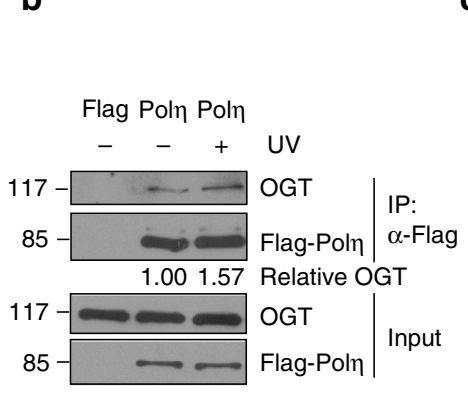

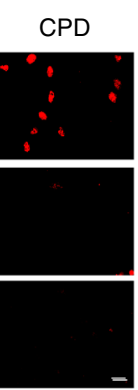

f

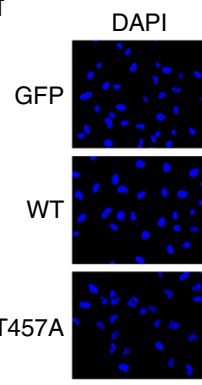

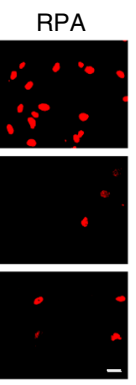

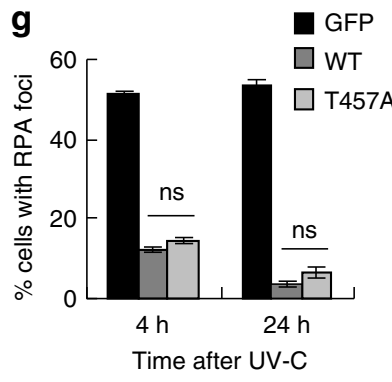

Fig. 2 T457A has no effect on the efficiency of Poln to bypass CPDs. a 293T cells transfected with GFP-Poln were treated with Thiamet-G and glucose. Cells were irradiated with UVC $\left(15 \mathrm{~J} \mathrm{~m}^{-2}\right.$ ) and harvested $4 \mathrm{~h}$ later (top panel) or at the indicated time points (bottom panel). The cell lysates were immunoprecipitated with GFP-Trap A followed by immunoblotting with O-GIcNAc and GFP antibodies. Experiment was repeated three times. SE short exposure, LE long exposure. b $293 \mathrm{~T}$ cells transfected with Flag-Poln were irradiated with UVC $\left(15 \mathrm{~J} \mathrm{~m}^{-2}\right)$ and harvested $4 \mathrm{~h}$ later. The cell lysates were immunoprecipitated and the relative IPed OGT was shown. Experiment was repeated twice. c U2OS cells transfected with indicated GFP-Poln constructs were UVC irradiated and incubated for $8 \mathrm{~h}$. The proportions of GFP-Poln expressing cells with $>30$ foci were determined by counting at least 200 cells in each experiment. d Poln-deficient XP30RO cells infected with GFP, WT or T457A GFP-Poln lentivirus were selected by flow cytometry. The levels of Pol $\eta$ protein in stable clones were examined with anti-Poln. Tubulin: loading control. e Representative images of cells stained with CPDs (red) in ssDNA of nuclei (DAPI, blue) after UV irradiation. XP30RO cells stably expressing GFP, WT or T457A GFP-Pol $\eta$ were irradiated with $5 \mathrm{~J} \mathrm{\textrm {m } ^ { - 2 }}$ UVC and cultured for 4 h. The cells were treated with $1 \%$ Triton-X100 for 2 min prior to fixation. Scale bars: $10 \mu \mathrm{m}$. $\mathbf{f}$ Representative images of cells stained with DAPI and RPA2 after UV irradiation. XP30RO cells stably expressing GFP, WT or T457A GFP-Poln were irradiated with $5 \mathrm{~J} \mathrm{~m} \mathrm{~m}^{-2} \mathrm{UVC}$ and further cultured. At the indicated time points, cells were treated with $0.5 \%$ Triton-X100 for 30 min followed by immunofluorescence. Scale bars: $10 \mu \mathrm{m}$. $\mathbf{g}$ Quantification of the percentage of cells with $>10$ RPA foci. For each cell line at each time point, at least 250 cells were counted. Data represent means \pm SEM from three independent experiments. ns not significant

effect (Fig. 3f). Hence, Poln T457A mutation impairs cellular response to UV radiation. We also determined whether depletion of OGT would affect cellular response to UV radiation. We found that knockdown of OGT in $293 \mathrm{~T}$ cells significantly abrogated Pol $\eta$ polyubiquitination (Supplementary Fig. 5a) and promoted UV light-induced mutagenesis (Supplementary Fig. 5b). Additionally, depletion of OGT led to an increased UV sensitivity in both U2OS and MRC5 cells (Supplementary Fig. 5c, d). These data support the notion that Poln O-GlcNAcylation at T457 is required for optimal cellular response to UV radiation.

T457A impairs Poln O-GlcNAcylation and ubiquitination. We next determined how T457A mutation could remarkably impair Pol $\eta$ polyubiquitination and promote its retention at replication forks. Based on the fact that Pol $\eta$ in Caenorhabditis elegans is subjected to $\mathrm{CRL} 44^{\mathrm{CDT} 2}$-dependent proteolysis in the context of chromatin-bound PCNA after DNA damage treatment ${ }^{50}$ and that the CRL4 ${ }^{\mathrm{CDT} 2}$ E3 ligase can induce ubiquitination of multiple PIP degron-containing proteins ${ }^{51}$, we speculated that CRL4 ${ }^{\mathrm{CDT} 2}$ might modulate human Poln polyubiquitination at replication forks and its subsequent degradation. In support of this, we found that depletion of the CRL4 ${ }^{\mathrm{CDT} 2}$ adaptor protein DDB1 in 293T cells led to an enhanced level of endogenous Poln (Fig. 4a) but a significant reduction in Pol $\eta$ polyubiquitination (Fig. 4b). To further explore whether Poln O-GlcNAcylation at T457 affects this process, both WT and T457A Poln protein levels were tested after silencing the components of CRL4 ${ }^{\mathrm{CDT} 2}$. As shown in Supplementary Fig. 6a, upon efficient reduction of either DDB1 or Cul4A, the level of WT but not T457A Poln was upregulated, suggesting that CRL4 ${ }^{\mathrm{CDT} 2}$ targets WT but not T457A Poln for polyubiquitination and subsequent proteolysis. In support, depletion of either DDB1 or CDT2, the components of CRL4 ${ }^{\mathrm{CDT} 2}$, remarkably decreased the polyubiquitination levels of WT but not T457A Pol $\eta$ in 293T cells (Fig. 4c, d). Additionally, a similar result was obtained when the chromatin-bound WT or T457A Poln were immunoprecipitated (Fig. 4e). To avoid nonspecific artifacts caused by aberrant p97-mediated removal of Pol $\eta$ from replication forks, p97 expression was knocked down and the polyubiquitination levels of chromatin bound WT and T457A Poln were compared. p97 depletion failed to change the trend of a significantly higher polyubiquitination level of WT Poln than that of T457A (Fig. 4f). Notably, p97 knockdown increased the polyubiquitination level of WT but not of T457A 
a

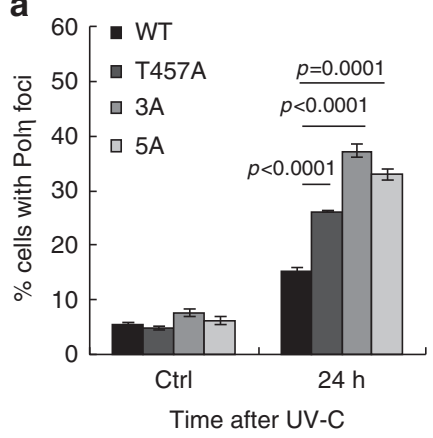

b

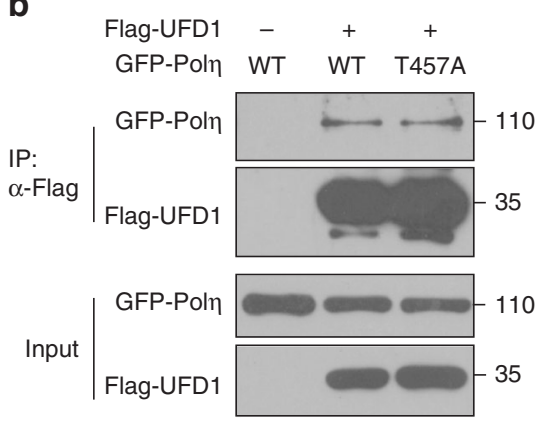

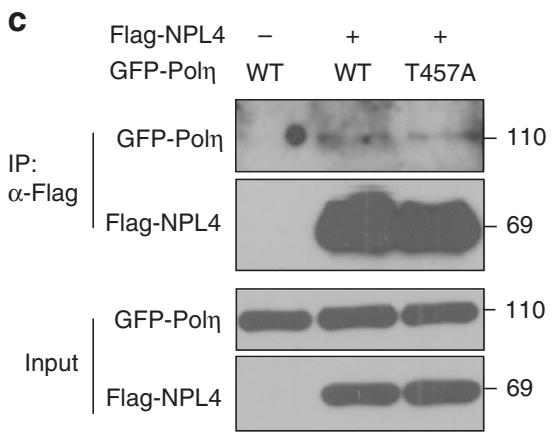

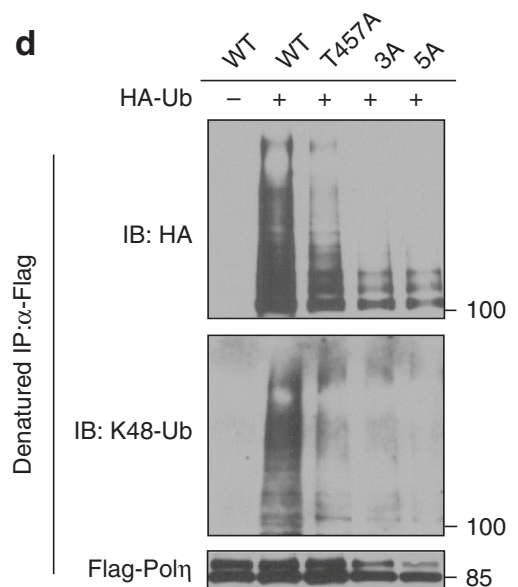

e

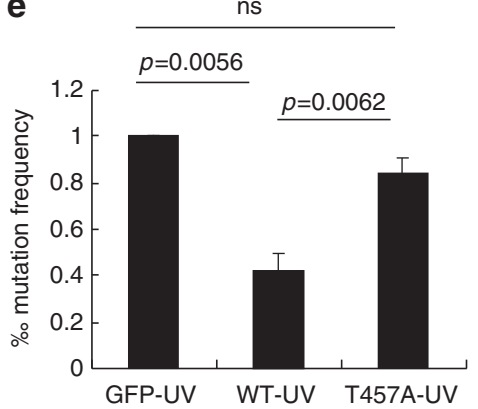

f

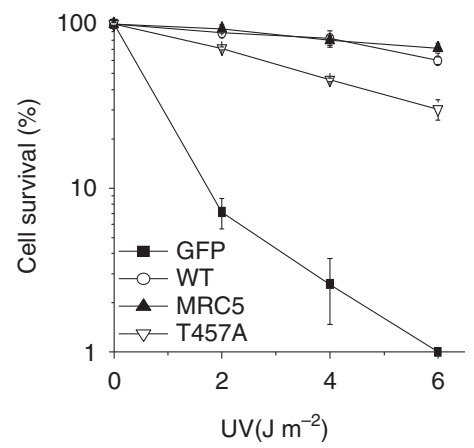

Fig. 3 T457A impairs Poln removal from chromatin after UV irradiation. a U2OS cells transfected with WT or mutated GFP-Poln constructs were irradiated with UVC $\left(15 \mathrm{~J} \mathrm{~m}^{-2}\right)$ and further incubated for $24 \mathrm{~h}$. The proportions of GFP-Poln-expressing cells with $>30$ foci were determined. Data represent means \pm SEM from three independent experiments. b, c Flag-UFD1 b or Flag-NPL4 c and GFP-Poln (WT or T457A) were transfected into 293 T cells. The lysates were immunoprecipitated using anti-Flag M2 beads. The inputs and immunoprecipitates were examined via western blotting using anti-GFP and anti-Flag antibodies. d 293T cells were transfected with WT or mutant Flag-Poln as well as HA-Ub or HA empty vector. The cell lysates were immunoprecipitated with anti-Flag M2 beads under denaturing condition, followed by immunoblotting with anti-HA, anti-K48-Ubiquitin, and anti-Flag antibodies. e Mutation frequency in damaged ( $400 \mathrm{~J} \mathrm{~m}^{-2}$ UVC) supF plasmid was determined. Data represent means \pm SEM from three independent experiments. ns not significant. $\mathbf{f}$ XP30RO cells stably expressing GFP, WT or T457A GFP-Poln and MRC5 cells were irradiated with the indicated doses of UVC and further incubated in medium supplemented with $0.4 \mathrm{mM}$ caffeine for 7-10 days. The number of cell clones was determined. Surviving fraction was expressed as a percentage of mock-treated cells. Experiment was repeated three times, giving similar results. The representative curve is shown. Error bar: $\mathrm{s} . \mathrm{d} ., n=3$

Poln (Fig. 4f). To support that O-GlcNAcylation at T457 promotes CRL4 ${ }^{\text {CDT2 }}$-mediated Poln polyubiquitination, we reconstituted the ubiquitination reaction in vitro. His-tagged WT or T457A Poln were co-expressed with GST-OGT in Escherichia coli cells, in which WT but not T457A Poln exhibited a strong OGlcNAcylation signal (Supplementary Fig. 6b, c). The purified WT or T457A His-Poln were then used as substrates for in vitro ubiquitination. Components of CRL4 ${ }^{\mathrm{CDT} 2}$ E3 ligase complex were co-expressed and immunopurified by anti-FLAG antibody and eluted with the FLAG epitope peptide (Supplementary Fig. 6d). The in vitro ubiquitination result showed that, without co-expression of OGT, WT and T457A Poln exhibited similar extent of ubiquitination (Supplementary Fig. 6e). After coexpression of OGT, the ubiquitination level of WT but not of T457A manifested a remarkably increase. These data suggested that Poln O-GlcNAcylation at T457 promotes CRL4 ${ }^{\mathrm{CDT} 2}$-mediated Pol $\eta$ polyubiquitination, which is the prerequisite for $\mathrm{p} 97$ modulated Poln removal from chromatin. Consistently, CDT2 depletion significantly delayed the dissociation of WT but not of T457A Poln from replication forks at $24 \mathrm{~h}$ after UV irradiation (Supplementary Fig. 6f). To further determine why CRL4 CDT2 preferentially polyubiquitinates WT instead of T457A Poln, we performed Co-IP experiments and found that T457A mutation significantly decreased the interactions between Pol $\eta$ and DDB1 or CDT2 (Fig. 4g, h), whereas it had no obvious effects on its binding with MDM2, another E3 ligase involved in Poln polyubiquitination $^{26}$ (Supplementary Fig. 6g). Therefore, the diminished polyubiquitination of T457A Pol $\eta$ is likely due to its reduced association with the E3 ligase CRL4 ${ }^{\mathrm{CDT} 2}$. We further performed in vitro pull down assay and found that, under the presence of GST-OGT, which promoted Poln O-GlcNAcylation, the association between Pol $\eta$ and DDB1 or CDT2 was significantly enhanced, suggesting that Poln O-GlcNAcylation promotes its interaction with CRL4 ${ }^{\mathrm{CDT} 2}$ complex (Supplementary Fig. 6h). Taken together, these results demonstrated that T457A mutation affects Poln O-GlcNAcylation and Poln association with CRL4 ${ }^{\mathrm{CDT} 2}$ and impairs $\mathrm{CRL} 4^{\mathrm{CDT} 2}$-mediated Pol $\eta$ polyubiquitination on chromatin, thereby limiting p97-dependent removal of Poln from replication forks upon completion of TLS.

T457A impairs Pol $\eta$ polyubiquitination at K462. Next, we attempted to identify the specific lysine residue(s) in Pol $\eta$, whose polyubiquitination is not only required for timely removal of Poln from replication forks but also can be abrogated by T457A mutation. We first analyzed the ubiquitination sites in WT and T457A Pol $\eta$ using quantitative MS. As shown in Supplementary Fig. 7a, b, T457A mutation markedly reduced both overall and 
a

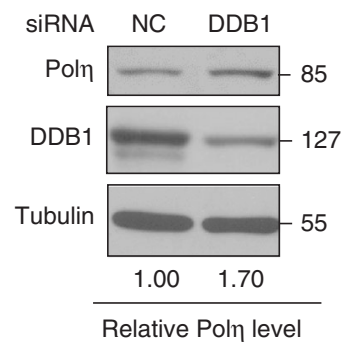

d
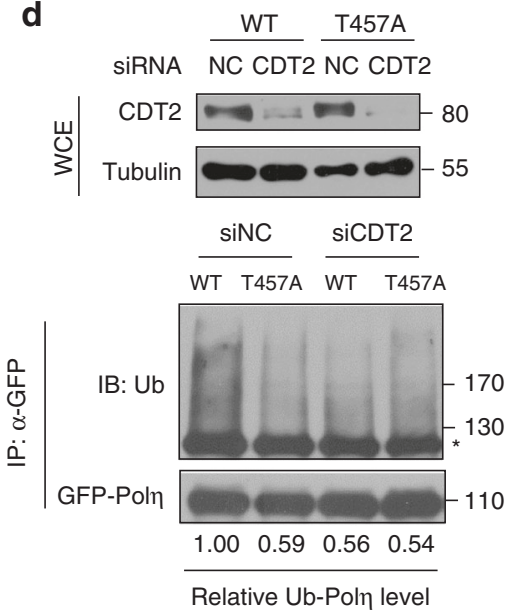

g

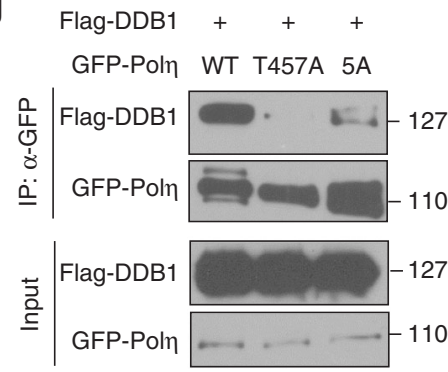

b

$$
\begin{array}{cccc}
\text { HA-Ub } & \multicolumn{2}{l}{\text { Flag-Poln }} & \text { Flag } \\
\text { SiRNA } & \text { NC } & \text { DDB1 } & \text { NC }
\end{array}
$$

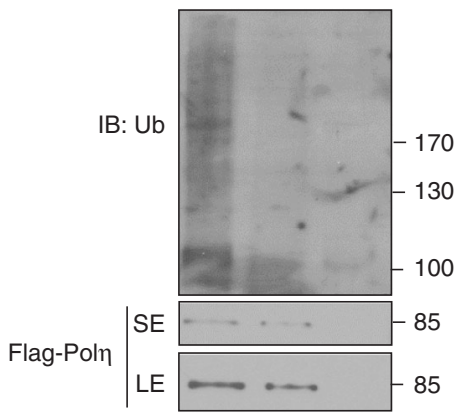

e
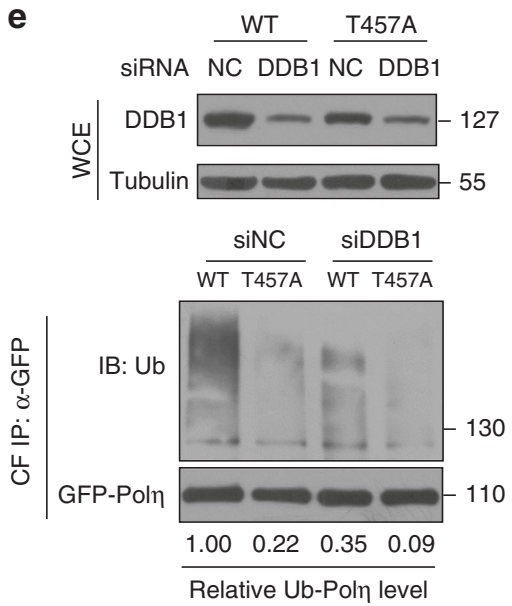

h

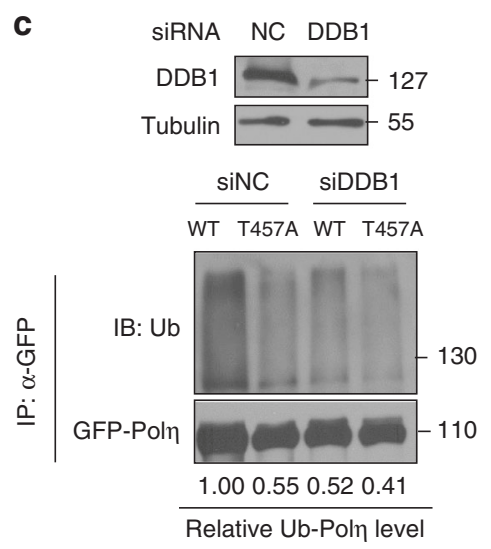

f
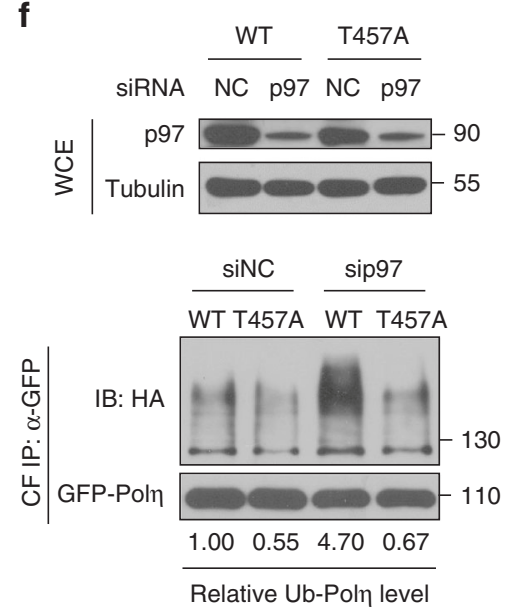

GFP-Poln $\frac{\text { Input(1/50) }}{\text { WT WT T457A }} \quad \frac{\text { IP: } \alpha \text {-Flag }}{\text { WT WT T457A }}$

Flag-CDT2 $-++\quad+++$

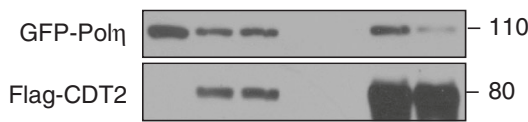

Fig. 4 T457A impairs CRL4 ${ }^{C D T 2}$-mediated Poln polyubiqitination. a 293T cells were transfected with siDDB1 or siNC oligos. Seventy-two hours later, the cells were harvested and the protein levels of Poln and DDB1 were detected by western blotting. Tubulin: loading control. b Flag empty vector or Flag-Pol $\eta$ and HA-Ub were transfected in siNC- or siDDB1-treated 293T cells. The cells lysates were immunoprecipitated by anti-Flag M2 beads and analyzed by immunoblotting with anti-Ubiquitin and anti-Flag antibodies. SE short exposure, LE long exposure. c, d WT or T457A GFP-Poln and HA-Ub were

transfected into siDDB1-treated $\mathbf{c}$ or siCDT2-treated $\mathbf{d} 293$ T cells. The lysates were immunoprecipitated using GFP-Trap A. The immunoprecipitates were analyzed via western blotting using anti-GFP and anti-Ubiquitin antibodies. The protein levels of DDB1 and CDT2 were detected by immunoblotting. WCE whole cell lysate. Tubulin: loading control. Asterisk denote non-specific signal. e WT or T457A GFP-Poln and HA-Ub were transfected into siDDB1-treated 293T cells. The chromatin fractions (CF) were harvested followed by immunoprecipitation with GFP-Trap A. The immunoprecipitates were analyzed as in c. f WT or T457A GFP-Poln and HA-Ub were transfected into p97 siRNA (sip97)-treated 293T cells. The chromatin fractions were immunoprecipitated and analyzed as in c. The p97 protein level was detected by western blot. $\mathbf{g}$ Flag-DDB1 and GFP-Poln (WT, T457A, or 5A) were transfected into 293 T cells. The lysates were immunoprecipitated using GFP-Trap A. The immunoprecipitates and inputs were examined via western blotting using anti-GFP and antiFlag antibodies. h Flag empty vector or Flag-CDT2 and GFP-Poln (WT or T457A) were transfected into 293T cells. The lysates were immunoprecipitated and analyzed as in $\mathbf{g}$

K48-linked ubiquitination levels of Poln, consistent with the result in Fig. 3d. Moreover, seven individual lysine residues (K131, K163, K311, K453, K462, K494, and K682) in the Poln protein were faithfully found to be ubiquitinated (Fig. 5a). Specifically, T457A mutation caused a significant decrease in ubiquitination level at residues K131, K453, and K494 and an even more pronounced reduction at K462 (Fig. 5a and
Supplementary Fig. 7c). Given that protein O-GlcNAc modification usually interplays with other PTMs at adjacent sites, we then focused on K453 and K462 in the vicinity of T457. We mutated K453 and K462 residues to arginine, respectively, to examine their effects on Poln removal after UV radiation. Interestingly, we found that K462R but not K453R mutation compromised the timely Poln disassembly, just analogous to 

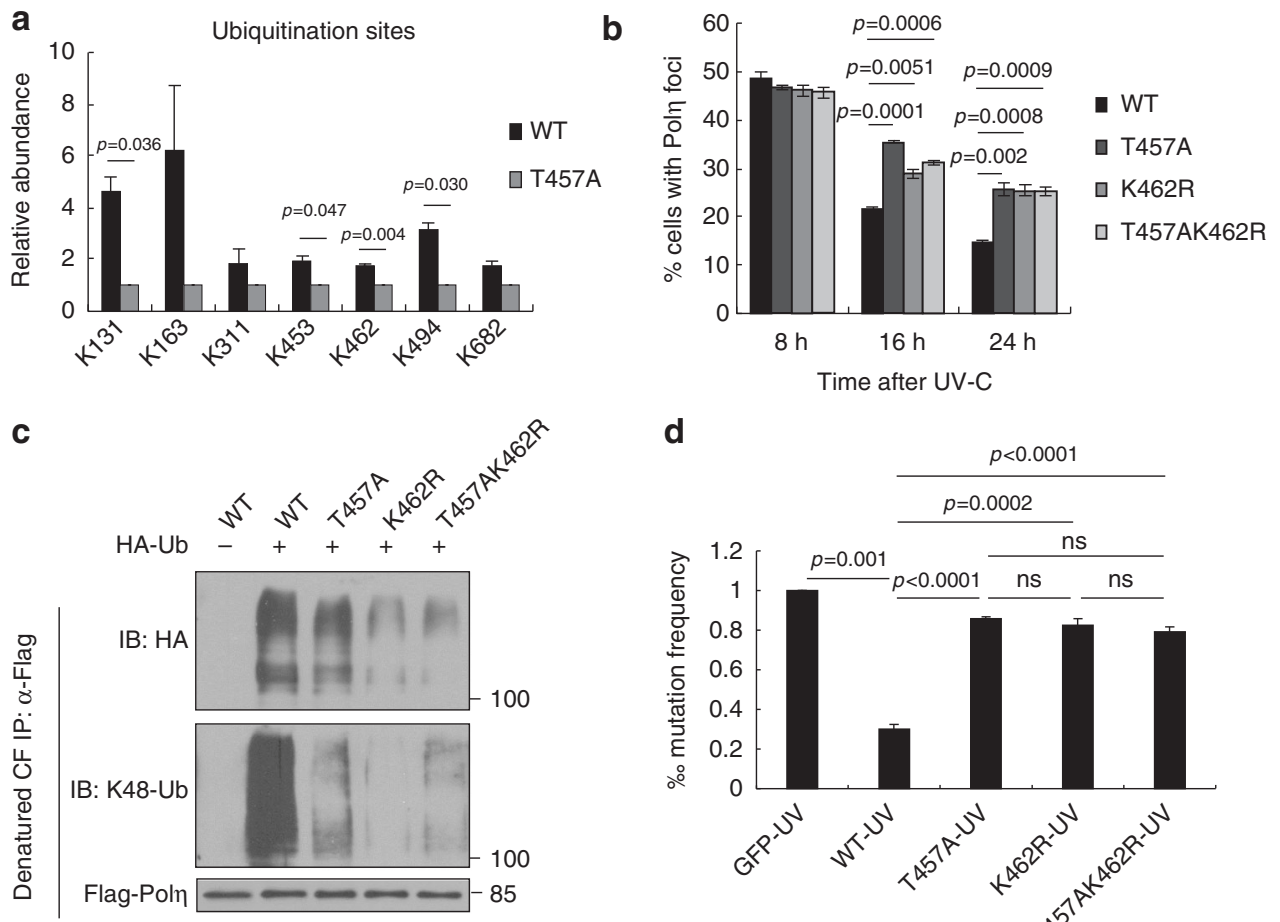

d

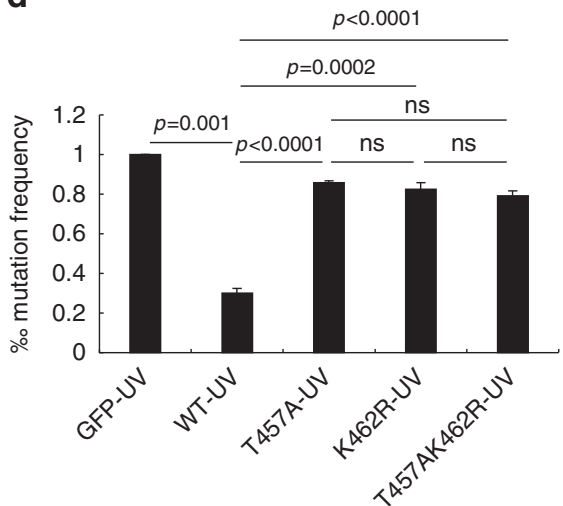

e

f
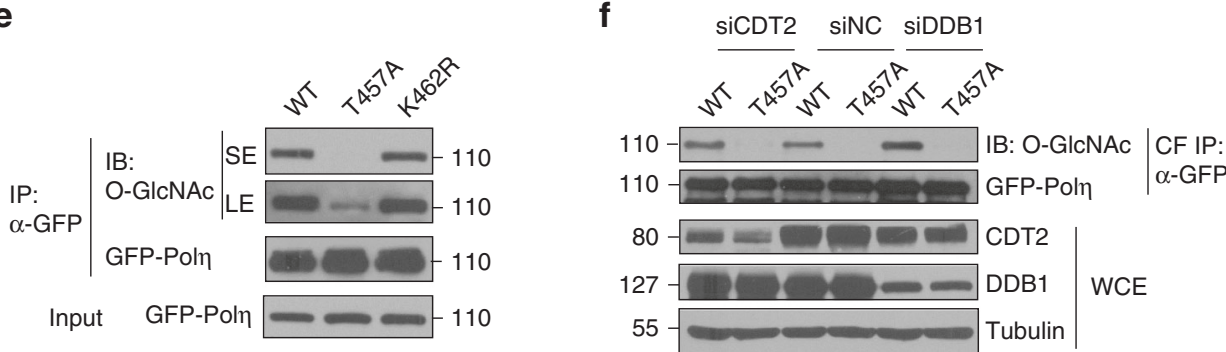

Fig. 5 T457A impairs Poln polyubiqitination at K462. a 293T cells transfected with Flag empty vector, WT, or T457A Flag-Poln and HA-Ub were immunoprecipitated with anti-Flag agarose in a denatured condition followed by quantitative MS analysis as described in "Methods" section. All ubiquitination sites identified in WT and T457A Poln. Error bars: s.e.m. b U2OS cells transfected with WT or mutated (T457A, K462R, and T457AK462R) GFP-Pol $\eta$ were irradiated by UVC $\left(15 \mathrm{~J} \mathrm{~m}^{-2}\right)$ and further incubated for $24 \mathrm{~h}$. The proportion of GFP-Pol $\eta$-expressing cells with $>30$ foci was determined. Data represent means \pm SEM from three independent experiments. c 293 T cells were transfected with WT or mutated (T457A, K462R, and T457A/ K462R) Flag-Poln and HA-Ub. The collected chromatin fractions (CFs) were denatured followed by immunoprecipitation with anti-Flag agarose. The immunoprecipitates were immunoblotted with anti-HA, anti-K48-Ubiquitin, and anti-Flag antibodies. $\mathbf{d}$ Mutation frequency in damaged (400 J $\mathrm{m}^{-2} \mathrm{UVC}$ ) supF plasmid was determined. Data represent means \pm SEM from three independent experiments. ns not significant. e WT, T457A, or K462R GFP-Poln were transfected into 293T cells. The cell lysates were immunoprecipitated with GFP-Trap A, followed by immunoblotting with anti-O-GlcNAc and anti-GFP antibodies. "SE" and "LE" represent short and long exposure, respectively. $f$ WT or T457A GFP-Poln were transfected into siDDB1-, siCDT2-, or siNC-treated 293T cells. The chromatin fractions were immunoprecipitated with GFP-Trap A and analyzed via western blotting with anti-O-GlcNAc and anti-GFP antibodies

T457A mutation (Fig. 5b and Supplementary Fig. 7d). Meanwhile, T457A/K462R double mutation did not cause a further delay in Poly dissociation from replication forks at 16 and $24 \mathrm{~h}$ after UV irradiation compared to either T457A or K462R single mutation (Fig. 5b), suggesting that K462 ubiquitination is downstream of T457 O-GlcNAcylation, one pathway critical in timely removal of Poln from replication forks.

K462 has been reported to be a potential CRL modification site identified in a systematic proteomic study ${ }^{52}$, which prompted us to assess whether K462 is a specific ubiquitination site catalyzed by the CRL4 ${ }^{\mathrm{CDT}}$ complex. After co-transfecting Poln with Flag-tagged CRL4 ${ }^{\mathrm{CDT} 2}$ adaptor protein DDB1, the polyubiquitination level of Poln was found to be significantly reduced when WT Poln was mutated to K462R (Supplementary Fig. 7e). Then we transfected HA-Ub with WT, T457A, K462R, or T457A/K462R Flag-Pol $\eta$ constructs into $293 \mathrm{~T}$ cells and collected the chromatin fractions for denatured IP. We observed that, similar as T457A, K462R mutation also significantly reduced the polyubiquitination of chromatin Poln (Fig. 5c). Using ubiquitin chain-specific K48 antibodies, we further confirmed that K462R mutation remarkably decreased Poln K48-polyubiquitination (Fig. 5c). Consistently, T457A/K462R double mutation did not induce further reduction in Poln K48polyubiquitination, supporting that K462 polyubiquitination probably sequentially occurs after T457 O-GlcNAc modification. Furthermore, K462R or T457A/K462R Poln-complemented XP30RO cells exhibited similar levels of UV-induced mutation frequency and UV hypersensitivity as T457A Polncomplemented XP30RO cells (Fig. 5d and Supplementary Fig. 7f). We also transfected WT, T457A, and K462R GFPPoln into 293T cells and compared the level of their O-GlcNAc modification. Unlike T457A, K462R mutation did not impair 
Poln O-GlcNAcylation (Fig. 5e), which is consistent with our hypothesis that $\mathrm{K} 462$ polyubiquitination happens after T457 OGlcNAcylation. In line with this, knockdown of the CRL4 CDT2 components did not impair Poln O-GlcNAcylation either (Fig. 5f).

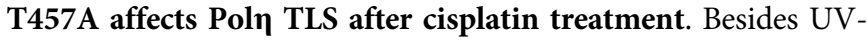
induced CPD lesions, Pol $\eta$ is also known to participate in cisplatin-induced TLS, which is believed to contribute to the development of cisplatin resistance ${ }^{12,19,21}$. We found that cisplatin treatment enhanced the interaction between OGT and Poln (Fig. 6a), and promoted Poln O-GlcNAcylation (Fig. 6b), suggesting that Poln O-GlcNAcylation regulates cellular response to cisplatin. As expected, the T457A-complemented cells displayed a much lower survival rate after cisplatin treatment when compared with WT Poln-complemented cells (Fig. 6c). We also examined Pol $\eta$ focus formation at different time points after cisplatin treatment. There are no significant differences in Pol $\eta$ focus formation at $24 \mathrm{~h}$ after cisplatin treatment between WT- and T457A-expressing cells (Fig. 6d). However, the reduction rate in proportion of GFP-Poln foci-positive cells at 34 and $46 \mathrm{~h}$ after drug removal was much slower in T457A-expressing cells than that of WT-expressing cells (Fig. 6d), indicating that T457A mutation might impair cellular TLS efficiency after cisplatin treatment through limiting Poln removal from damage sites. To test this possibility, we compared the RPA signals in WT and T457A Poln-complemented XP30RO cells after cisplatin

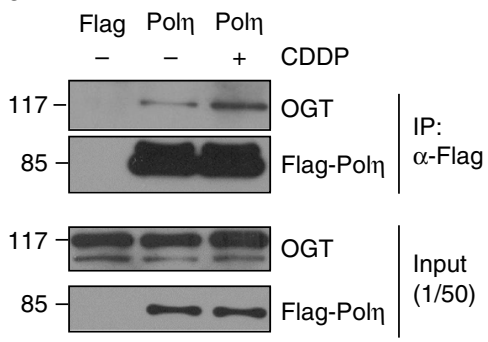

b

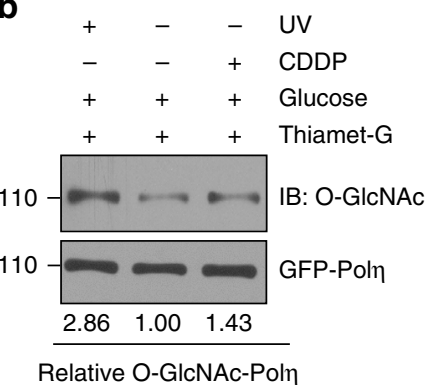

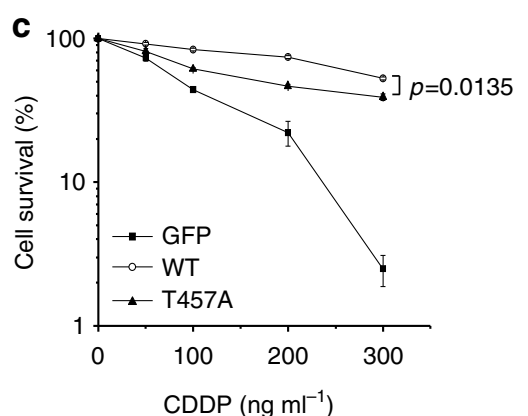

$\mathbf{f}$

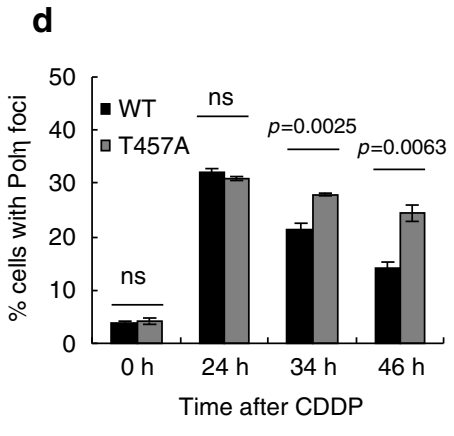

e
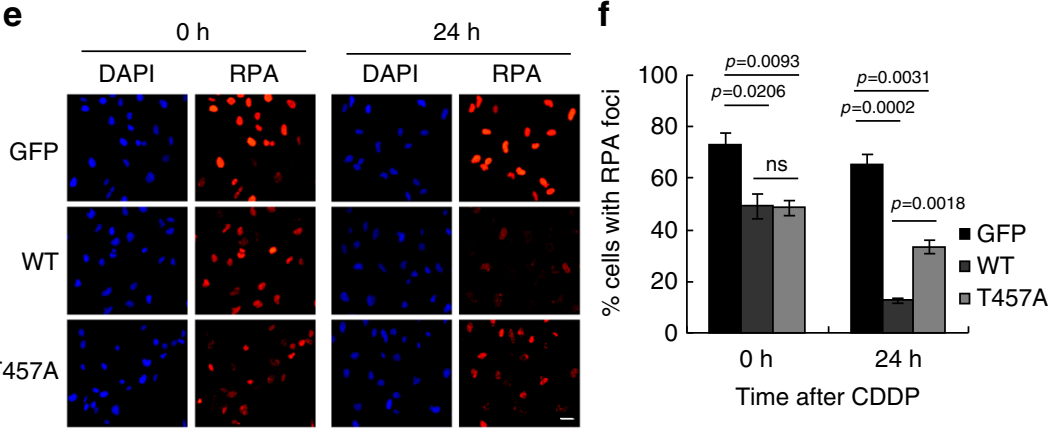

g

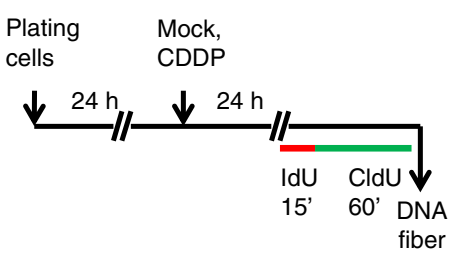

CTRL

CDDP

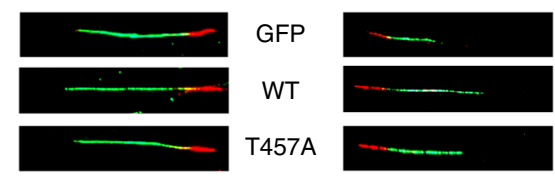

h

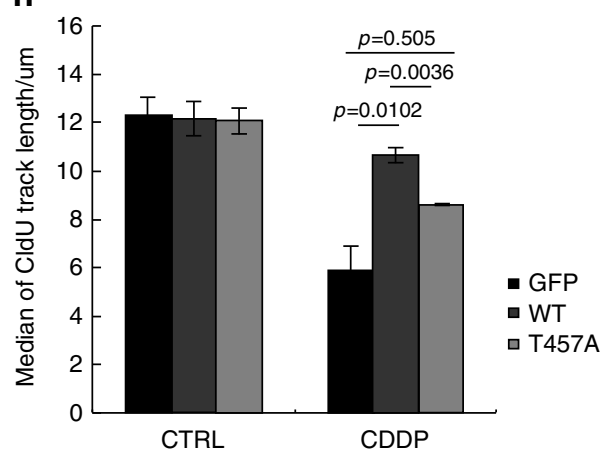

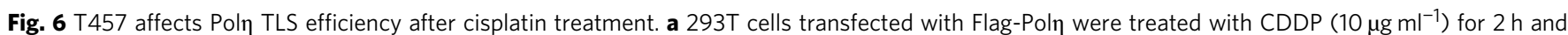
changed to drug-free media for $2 \mathrm{~h}$. The cell lysates were harvested for immunoprecipitation assay as in Fig. 1e. b $293 \mathrm{~T}$ cells transfected with GFP-Pol $\eta$ were treated with Thiamet-G and glucose. Cells were treated with UVC $\left(15 \mathrm{~J} \mathrm{~m}^{-2}\right)$ irradiation or cisplatin $\left(10 \mu \mathrm{g} \mathrm{ml} \mathrm{m}^{-1}\right)$ and harvested at 4 or $2 \mathrm{~h}$ later, respectively. The cell lysates were immunoprecipitated with GFP-Trap A and analyzed with anti-O-GlcNAc and anti-GFP antibodies. c XP3ORO cells stably expressing GFP, WT, or T457A GFP-Poln were treated with cisplatin for $24 \mathrm{~h}$ and further incubated for 7-10 days. Cell survival was analyzed as in Fig. 3f. d U2OS cells transfected with WT or T457A GFP-Poln were treated with cisplatin $\left(5 \mu \mathrm{g} \mathrm{ml}^{-1}\right)$ for $2 \mathrm{~h}$ followed by incubation with fresh media. At the indicated time points, the proportion of GFP-Poln expressing cells with $>30$ foci was determined. e Representative images of cells stained with DAPI and RPA2 after cisplatin treatment. XP30RO cells stably expressing GFP, WT, or T457A GFP-Poln were treated with cisplatin for $24 \mathrm{~h}$ and further cultured. At the indicated time points, cells were immunostained with anti-RPA2 antibody. Scale bars: $10 \mu \mathrm{m}$. $\mathbf{f}$ Quantification of the percentage of cells with $>10$ RPA2 foci. $\mathbf{g}$ Scheme of experimental design for replication fork progression in XP30RO cells stably expressing GFP, WT, or T457A GFP-Poln. After cisplatin $\left(1 \mu \mathrm{gll}^{-1}\right)$ treatment for $24 \mathrm{~h}$, representative DNA fibers were shown. $\mathbf{h}$ Median lengths of nascent replication tracts (labled with CldU) were given. Data represent means \pm SEM from three independent experiments. ns not significant 
treatment. The percentages of cells with RPA foci were similar between WT- and T457A-complemented cells immediately after cisplatin treatment (Fig. 6e, f). However, after $24 \mathrm{~h}$ recovery, the proportion of RPA foci-positive cells in WT Poln-expressing group (12.6\%) was significantly less than that in the T457A mutant-expressing group (33.4\%), supporting that T457A mutation impairs cisplatin-induced cellular TLS efficiency. We further performed DNA fiber analysis to investigate the direct effect of T457A mutation on bypass of cisplatin-induced DNA lesions in vivo. Cisplatin-treated cells were pulse-labeled with iododeoxyuridine (IdU) for $15 \mathrm{~min}$ followed by chlorodeoxyuridine CIdU for $60 \mathrm{~min}$ (Fig. 6g). Then the length of newly synthesized DNA strands (green fluorescent CIdU) was monitored. In the absence of cisplatin, the median fiber lengths in GFP, WT, or T457A Poln-expressing cell lines were similar (12.30, 12.17, and $12.07 \mu \mathrm{m}$, respectively; Fig. $6 \mathrm{~g}, \mathrm{~h}$ ), indicating that their replication fork rates were comparable. But after exposure to cisplatin, the median fiber length in T457A Poln-expressing cells $(8.62 \mu \mathrm{m})$ was substantially shorter than that in WT Poln-expressing cells (10.65 $\mu \mathrm{m})(p=0.0036)$, although it was longer than that in GFPcomplemented cells $(5.90 \mu \mathrm{m} ; p=0.505)$. These data provide direct evidence that inactivation of Poln O-GlcNAcylation at T457 impairs replication fork progression in vivo following cisplatin treatment.

\section{Discussion}

Poln is the only known DNA polymerase that has an enlarged active site to accommodate the entire CPD or Pt-GG for the $3^{\prime}$ base to instruct correct nucleotide incorporation ${ }^{19}$. It can be recruited to stalled replication forks through interaction with mUb-PCNA ${ }^{7,9,10,23}$. Owing to its low fidelity on undamaged DNA, timely removal of Poln from replication forks when it is not required is presumably essential for genome stability ${ }^{6}$. Although Poln-reduced affinity for the DNA beyond the CPD might contribute to its dissociation after $\mathrm{TLS}^{28}$, deubiquitination of mUb-PCNA by USP $1^{29}$ or USP $10^{30}$ can promote Poln displacement from replication forks. Notably, mUb-PCNA usually persists for many hours even after clearance of the damage and TLS polymerase foci ${ }^{31}$. Additionally, upon cisplatin exposure, efficient bypass of Pt-GG usually needs a second TLS polymerase to carry out primer extension after dCTP incorporation opposite the cross-linked bases by Pol $\eta^{6,19,53}$. This polymerase-switching between insertion and extension polymerases usually happens in the presence of mUb-PCNA. At present, how disassembly of Poln occurs in the persistence of mUb-PCNA still remains enigma.

Herein we demonstrated that human Poln undergoes OGlcNAcylation. Through MS, we showed that Poln is mainly OGlcNAcylated at residue T457. By monitoring the disappearance of CPDs in ssDNA ${ }^{41}$ and the disappearance of RPA foci ${ }^{44}$ after UV irradiation, we showed that T457A mutation does not affect the ability of Poln to bypass CPD lesions. Intriguingly, this mutation remarkably impairs disassembly of Poln from replication forks after TLS is finished, causing increased mutation frequency as well as UV sensitivity.

Considering that the p97-UFD1-NPL4 complex acts as a K48 ubiquitin-chain-dependent protein segregase to remove DNAbinding proteins at the sites of DNA damage ${ }^{54}$, it is therefore tempting to speculate that O-GlcNAcylation at T457 is likely required for timely Poln ubiquitination and thereby p97dependent dissociation from replication forks after TLS. Consistently, we found that Poln interacts with the CRL4 ${ }^{\mathrm{CDT} 2}$ complex, which is responsible for ubiquitination of multiple PIP degron-containing proteins ${ }^{51}$. Inactivation of Poln OGlcNAcylation at T457 inhibits its association with this E3 complex leading to a distinct reduction in the level of Poln K48- polyubiquitination. Additionally, based on quantitative MS analysis, several potential Pol $\eta$ ubiquitination sites regulated by T457 O-GlcNAcylation were identified. Among them, two T457adjacent residues (K453 and K462) were mutated to assess their effects on Pol $\eta$ removal from replication forks. Only K462R manifested an epistasis relationship with T457A in restraining timely Poln dissociation from replication forks as well as causing an increased rate of mutagenesis and hypersensitivity after UV irradiation. Further analysis revealed that K462 can be polyubiquitinated through K48-linked ubiquitin chains on chromatin, echoing the previous report ${ }^{52}$ that $\mathrm{K} 462$ is a potential CRL modification site. Interestingly, synergistic effects on reduction of Poln K48-linked polyubiquitination were not observed in the T457A/K462R double mutant compared with the K462R single mutant, suggesting that K462 polyubiquitination sequentially happens after T457 O-GlcNAc modification. In support of this, K462R mutation or depletion of the components of CRL4 ${ }^{\mathrm{CDT} 2} \mathrm{E} 3$ ligase does not impair Poln O-GlcNAcylation at T457.

Besides CPDs, Pol $\eta$ is capable of replicating across Pt-GG adducts $^{6,18,19}$, which is believed to contribute to the development of cisplatin resistance ${ }^{12,19,21}$. We demonstrated an augmentation of Poln O-GlcNAcylation after cisplatin treatment, and inactivation of this modification due to T457A mutation sensitizes cells to cisplatin killing. Interestingly, cisplatin-induced RPA foci disappear more slowly in cells carrying T457A Poln compared to the WT counterparts, suggesting a TLS defect upon inactivation of Poln O-GlcNAcylation at T457. Mechanistically, through DNA fiber analysis, we confirmed that TLS is strongly reduced across cisplatin-damaged DNA after T457A mutation. This impact seems distinct from the effect of T457A mutation on TLS across CPDs. However, in light of the fact that Poln can finish bypass synthesis of CPD by its own, but fail to support complete bypass of Pt-GG ${ }^{19}$, it is likely that the inability of T457A Poln to fulfill efficient TLS after cisplatin treatment might also be attributed to its restrained disassociation from replication forks.

In our study, we also showed that Poln O-GlcNAcylation is induced by both UV and cisplatin exposure. Given that OGT and O-GlcNAcylation can be enriched at the sites of DNA damage ${ }^{38}$, it is conceivable that the upregulation of Poln O-GlcNAcylation occurs on chromatin, after it is recruited to stalled replication forks. Congruently, inactivation of Poln O-GlcNAcylation by T457A mutation does not impair Poln focus formation after UV and cisplatin treatment. Therefore, we speculate that a key function of Poln O-GlcNAcylation at T457 is to facilitate polymerase switching during TLS-mediated bypass of fork-blocking lesions and additionally help to dissociate low-fidelity TLS polymerases from replication forks after TLS, thereby restricting excessive mutagenesis (Fig. 7). This mechanism is apparently different from that of a recently identified phosphorylation of serine 687 on Pol $\eta$, which is postulated to facilitate Pol $\eta$ departure from PCNA through bringing negative charges to the nuclear localization signal of Pol $\eta^{55}$.

Recently, Pol $\eta$ sumoylation at K163 was identified, which was reported to be required to prevent under-replicated $\mathrm{DNA}^{56}$. Interestingly, through MS, we found that K163 residue can also be ubiquitinated, which is downregulated by T457A mutation. Therefore, it is yet to be determined how these different PTM modes interplay to collaboratively regulate Pol $\eta$ in vivo.

The upregulation of O-GlcNAcylation has been recognized as a common feature of cancer cells ${ }^{57}$. Recently, Pol $\eta$ is reported to play a vital role in improving ovarian cancer survival by enrichment of cancer stem cells after cisplatin treatment ${ }^{21}$. Based on our result that mutation of the major Poln O-GlcNAcylation residue T457 sensitizes cell to cisplatin exposure, it is reasonable to speculate that targeting Pol $\eta \mathrm{O}-$ GlcNAcylation in ovarian cancer cells may have potential therapeutic benefits. Since Pol $\eta$ can carry 

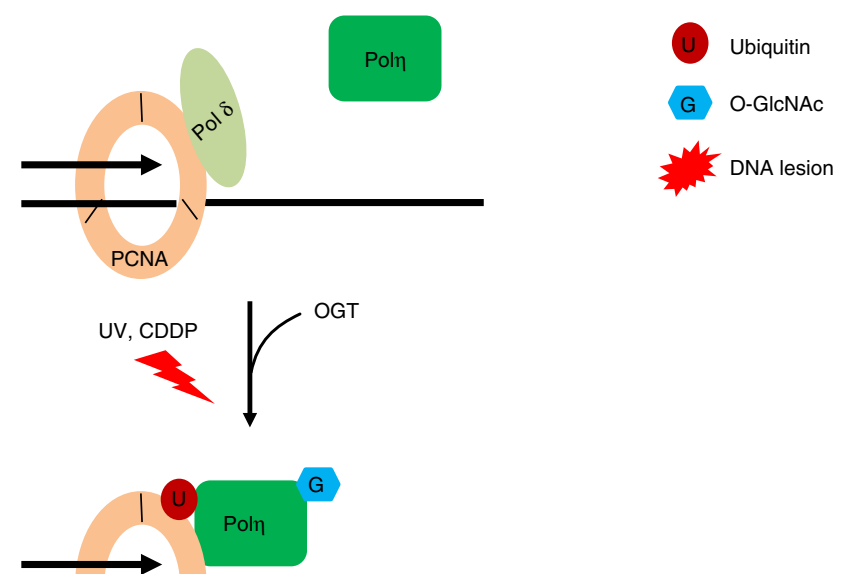

O-GlcNAcylation
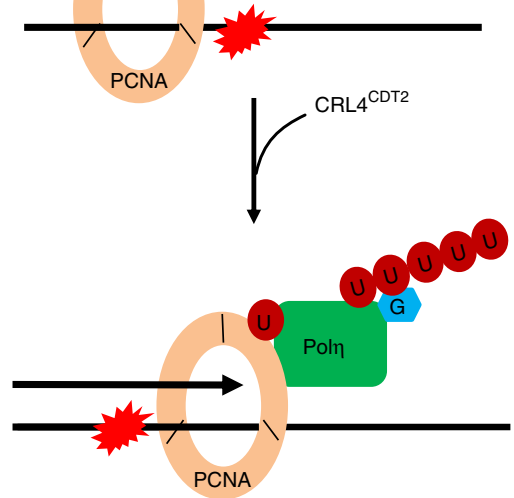

Ubiquitination

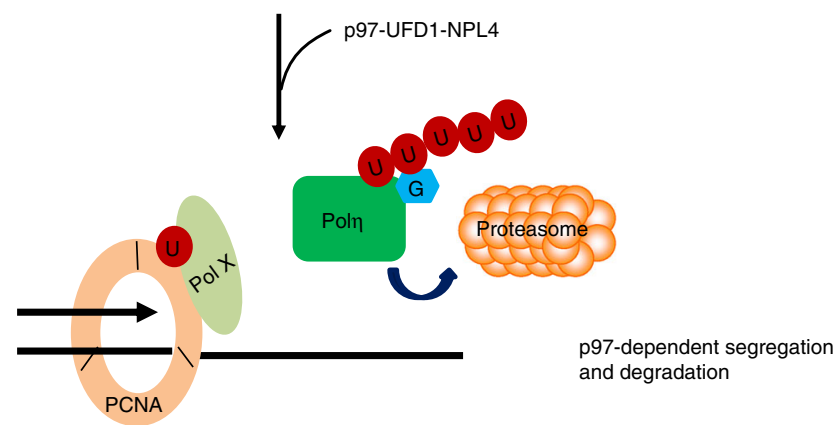

Fig. $7 \mathrm{~A}$ proposed model depicting the role of Poln O-GlcNAcylation in its removal after TLS. Upon UV or CDDP exposure, Poln is recruited to stalled replication forks and gets O-GIcNAcylated by OGT. Once Poln's role in TLS is completed, O-GIcNAcylated Poln is ubiquitinated by CRL4 ${ }^{\mathrm{CDT} 2}$ E3 ligase complex. The polyubiquitinated Poln is then recognized by p97-UFD-NPL4 complex, resulting in its dissociation from replication forks and degradation

out other roles beyond TLS in vivo ${ }^{58-61}$, it will be of great value to examine whether Poln O-GlcNAcylation also affects these processes. Additionally, considering that OGT and O-GlcNAcylation can be enriched at the sites of DNA damage ${ }^{38}$, where other TLS polymerases and multiple other DDR factors can be recruited, it will be interesting to delineate whether O-GlcNAcylation also modulates the dynamic association/dissociation of those factors from the sites of damage in future studies.

In summary, our results reveal that loss of O-GlcNAcylation at T457 in Poln significantly decreases K48-linked polyubiquitination at adjacent K462, thereby restraining its timely removal from replication forks and subsequent polymerase switching during and after TLS. Given the key role of Poln in TLS and cellular resistance to chemotherapy, O-GlcNAcylation adds a further layer of regulation that controls TLS and genome stability in vivo.

\section{Methods}

Plasmids and reagents. Human Poln cDNA was cloned in pEGFP-C3 (Clontech) or pCMV-2xFlag-SBP (modified from pCMV-3xFlag-myc) to generate eGFP or Flag fusion proteins. Different O-GlcNAc mutants including T457A, 3A (T457A, S458A, S459A), 5A (T457A, S458A, S459A, S466A and T471A), K453R, K462R, and T457A/K462R were constructed based on pEGFP-C3-Poln or pCMV-2xFlagSBP-Pol $\eta$ by site-mutated PCR amplification. Flag-NPL4 and Flag-UFD1 were gifts from Dr. Wei Li (Institute of Zoology, Chinese Academy of Science). Flag-CDT2 and Myc-Cul4A were gifts from Dr. Hengyu Fan (Zhejiang University). pcDNA3Flag-DDB1 was purchased from Addgene. pET-28a-Poln was a gift from Dr. Wei Yang (NIDDK, National Institutes of Health). For generating internal deletion mutants, cDNA fragments of Poly or OGT genes were PCR amplified and cloned into pCMV-3xFlag or pCMV-Myc, respectively. Anti-Flag M2 agarose affinity gel (A2220) was purchased from Sigma (St Louis, MO). GFP-Trap A beads (gta-20) were from Chromotek. Antibodies sources were as follows: mouse anti-Flag (F1804, 1:1000) from Sigma (St Louis, MO); anti-CPD (CAC-NM-DND-001, 1:4000) from Cosmo Bio Co (Tokyo, Japan); anti-Poln (ab17725, 1:1000) from Abcam and Dr. Simone Sabbioneda (Istituto di Genetica Molecolare, Italy); antiRPA32 (ab2175, 1:800), p97 (ab11433, 1:2000), and O-linked $\beta-\mathrm{N}$ acetylglucosamine (O-GlcNAc, ab2739, 1:2000) from Abcam; anti-HA (902302, 1:2000) from BioLegend; anti-Myc (MMS-150R-500, 1:1000) from Covance; antiHis (HT501-02, 1:1000) from Beijing TransGen Biotech Co., Ltd; anti-H3.1 (P30266, 1:2000) from Abmart; anti- $\beta$-Tubulin (AbM59005-37-PU, 1:4000) from Beijing Protein Innovation (Beijing, China); anti-CDT2 (A300-948A, 1:500) and Cullin 4A (A300-739A, 1:1000) from Bethyl; anti-DDB1 (NB100-625, 1:1000) from Novus Biologicals; anti-GFP (sc-8334, 1:500), OGT (sc-32921, 1:1000), and ubiquitin (sc-8017, 1:1000) from Santa Cruz Biotechnology. Ubiquitin chain-specific Lys48 antibody $(4298,1: 200)$ was from CST (Cell Signaling Technology). Alexa Fluor-conjugated secondary antibodies were from Invitrogen. Uncropped immunoblots of the main figures are shown in Supplementary Figs. 8-10.

Cell culture and reagents. Human U2OS and 293T cells were obtained from the American Type Culture Collection (Rockville, MD). SV40-transformed MRC5 and XP30RO cells were kindly provided by Dr. Alan Lehmann ${ }^{5}$. The XP30RO-Poln cell line (a Pol $\eta$-deficient XP30RO cell line to express SFB-tagged Poln under the control of a tetracycline-inducible promoter) was kindly provided by Dr. Jun Huang ${ }^{42}$. These cell lines were grown in Dulbecco's modified Eagle's medium medium supplemented with $10 \%$ fetal bovine serum. All cells were grown at $37^{\circ} \mathrm{C}$ in the presence of $5 \% \mathrm{CO}_{2}$ if not specified. All cells were tested for mycoplasma contamination using the Lonza Mycoplasma Kit. For transient transfection experiments, cells were transfected with the indicated constructs, using Vigofect (Vigorous Biotechnology) following the manufacturer's protocols. For RNAi experiments, cells were transfected with small interfering RNAs (siRNAs) purchased from GenePharma (Shanghai, China) using RNAiMAX (Invitrogen) according to the manufacturer's instruction and analyzed $72 \mathrm{~h}$ later. The genespecific target sequences were as follows: DDB1-1 (GCAAGGACCUGCUGUUUAU), DDB1-2 (GGCCAAGAACAUCAGUGUG), CDT2 (GAAUUAUA CUGCUUAUCGA), p97-1 (CCCAAGAUGGAUGAAUUGCAGUUGU), p97-2 (AACAGCCAUUCUCAAACAGAA), Cul4A (GAAGCUGGUCAUCAAGAAC), OGT-1 (UAAUCAUUUCAAUAACUGCUUCUGC), and OGT-2 (GAUUAAGCCUGUUGAAGUC). The negative control (siNC) sequence (UUCUCCGAACGUGUCACGU) was also obtained from GenePharma.

Establishment of Poln rescue cell lines. Lentiviral vectors expressing a series of GFP-tagged Poln were constructed and transfected into 293T cells with packaging plasmids pCMV-DR and pMD2-VSVG to produce viral particles ${ }^{62}$. The lentiviral particles were then used to infect XP30RO cells with the addition of $8 \mu \mathrm{g} \mathrm{ml}^{-1}$ polybrene (Sigma-Aldrich). The stable cells were selected by flow cytometry, and individual clones were isolated. The expression levels of Poln were confirmed by western blotting with antibodies against GFP or Poln, as indicated.

Higher-energy collision dissociation MS. HEK293T cells expressing 2xFlag-Poln were incubated with $5 \mu \mathrm{M}$ Thiamet-G for $24 \mathrm{~h}$ and $30 \mathrm{mM}$ glucose for $3 \mathrm{~h}$. Cells were harvested and lysed with HEPES buffer $(50 \mathrm{mM}$ HEPES pH 7.5, $150 \mathrm{mM} \mathrm{NaCl}, 1$ mM EDTA, 1 mM EGTA, 10\% glycerol, 1\% Triton X-100, $25 \mathrm{mM} \mathrm{NaF,} 10 \mu \mathrm{M}$ $\mathrm{ZnCl}_{2}$ ). The whole-cell lysates were subjected to IP using anti-Flag M2 agarose. The bound proteins were eluted with $0.1 \mathrm{M}$ Glycine $(\mathrm{pH} 2.5)$ and neutralized by adding Tris ( $\mathrm{pH}$ 8.5). The elutions were precipitated using ice-cold acetone and resuspended in $8 \mathrm{M}$ urea and $100 \mathrm{mM}$ Tris ( $\mathrm{pH} 8.5$ ). After reduction with TCEP (Tris(2-Carboxyethyl)-Phosphine) ( $5 \mathrm{mM}$ ) for $20 \mathrm{~min}$ and alkylation with iodoacetamide (10 $\mathrm{mM}$ ) for $15 \mathrm{~min}$ at room temperature, the samples were diluted to $2 \mathrm{M}$ urea with $100 \mathrm{mM}$ Tris (pH 8.5), and each divided into four aliquots for Trypsin, Glu-C, or Elastase digestion $\left(1: 50\right.$ enzyme:substrate, $25^{\circ} \mathrm{C}$ for Glu-C and $37^{\circ} \mathrm{C}$ for Trypsin and Elastase) overnight. The digestions were quenched with $5 \%$ formic acid and pooled together. The LC-MS/MS analysis was performed on an Easy-nLC 1000 UPLC (Thermo Fisher Scientific) coupled to a Q Exactive mass spectrometer (Thermo Fisher Scientific). Peptides were loaded on a precolumn ( $75 \mu \mathrm{m}$ internal diameter, 8 $\mathrm{cm}$ long, packed with ODS-AQ $12 \mathrm{~nm}-10 \mu \mathrm{m}$ beads from YMC Co., Ltd), and separated on an analytical column $(75 \mu \mathrm{m}$ internal diameter, $11 \mathrm{~cm}$ long, packed with 
Luna C18 $3 \mu \mathrm{m}-100 \AA ̊$ resin from Phenomenex) using an acetonitrile gradient from 0 to $30 \%$ in $55 \mathrm{~min}$ and $30-80 \%$ in another $10 \mathrm{~min}$ at a flow rate of $300 \mathrm{nl} \mathrm{min}^{-1}$. Spectra were acquired in a data-dependent mode: the 15 most intense precursor ions from each full scan (Resolution 70,000) were isolated for HCD MS2 (Resolution $17,500)$ at NCE 27 with a dynamic exclusion time of 20 s. Precursors with $1+$ or unassigned charge states were excluded. For peptide identification, the MS2 spectra were searched against an EPI-IPI human database (forward and reversed sequences) using Prolucid with $50 \mathrm{ppm}$ mass accuracy for both precursor and fragment ions, with carbamidomethylation on cysteine as fixed modification and GlcNAc (203.079373) on serine, threonine, or tyrosine as differential modification ${ }^{63}$. Search results were filtered using DTASelect 2.0 with a $5 \%$ false discovery rate cutoff at the spectral level ${ }^{64}$. The GlcNAc-modified peptide spectrum was labeled using pLabel, requiring $20 \mathrm{ppm}$ mass accuracy for fragment ions ${ }^{65}$.

Chromatin fraction isolation. Cells were lysed in CSK-100 buffer $(100 \mathrm{mM} \mathrm{NaCl}$, $300 \mathrm{mM}$ sucrose, $3 \mathrm{mM} \mathrm{MgCl}$, $10 \mathrm{mM}$ PIPES pH 6.8, 1 mM EGTA, 0.2\% Triton $\mathrm{X}$-100) containing protease inhibitors at $4^{\circ} \mathrm{C}$ for $15 \mathrm{~min}$. Chromatin-associated proteins were released from the pellets by treatment with lysis buffer $(50 \mathrm{mM}$ HEPES pH 7.5, $50 \mathrm{mM} \mathrm{NaCl}, 0.05 \%$ SDS, $2 \mathrm{mM} \mathrm{MgCl}_{2}, 10 \%$ Glycerol, $0.1 \%$ Triton $\mathrm{X}-100,10$ units of Benzonase Nuclease) containing protease inhibitors at $4{ }^{\circ} \mathrm{C}$ overnight. The supernatants were separated by SDS-PAGE and detected by immunoblotting with the indicated antibodies ${ }^{9}$.

Immunofluorescence. Cells were cultured on glass coverslips. Briefly, cells were treated with $0.5 \%$ Triton X-100 for $5-30$ min before fixing in $4 \%$ paraformaldehyde. Then the cells were incubated with $5 \%$ fetal bovine serum and $1 \%$ goat serum for $1 \mathrm{~h}$ followed by incubation with anti-RPA2 for $45 \mathrm{~min}$. After staining with secondary antibodies (Alexa Fluor 568; Molecular Probes) for $45 \mathrm{~min}$, cover slips were mounted in Vectashield mounting medium (Vector Laboratories) containing the nuclear stain 4,6-diamidino-2-phenylindole (DAPI). Images were acquired with a Leica DM5000 (Leica) equipped with HCX PL S-APO $63 \times 1.3$ oil CS immersion objective (Leica) and processed with Adobe Photoshop 7.0 $0^{41}$

For quantitative analysis of UV-induced Poln focus formation, U2OS cells transfected with GFP-Pol $\eta$ were treated with UVC $\left(15 \mathrm{~J} \mathrm{~m}^{-2}\right)$ and fixed with $4 \%$ paraformaldehyde $8-24 \mathrm{~h}$ later after UV irradiation ${ }^{41}$. Images were acquired using a Leica DM5000 (Leica) equipped with HCX PL S-APO $63 \times 1.3$ oil CS immersion objective (Leica). A minimum of 200 nuclei was analyzed for each treatment.

Detection of unreplicated CPDs in UV-treated cells. Detection of CPDs in single-stranded DNA templates was performed as previously described ${ }^{41}$ with some modifications. Briefly, cells cultured on coverslips were irradiated with 0 or 5 $\mathrm{J} \mathrm{m}^{-2}$ UVC light. Four hours later, cells were treated with $1 \%$ Triton-X100 in phosphate-buffered saline (PBS) for 2 min and subsequently fixed in $2 \%$ Formaldehyde/PBS containing $0.5 \%$ Triton-X100 for $15 \mathrm{~min}$ at room temperature. Unreplicated photoproducts were identified in non-denatured DNA using primary mouse monoclonal antibody against CPDs (TDM2, CosmoBio). After incubation with secondary Alexa Fluor 568-labeled goat-anti-mouse antibodies (Molecular Probes, Inc.), nuclei were stained with DAPI. Images were analyzed by fluorescent microscopy.

Cell cycle analysis. U2OS cells transfected with WT or T457A GFP-Pol $\eta$ were treated with UVC $\left(15 \mathrm{~J} \mathrm{~m}^{-2}\right)$ and harvested by trypsinization $24 \mathrm{~h}$ later after UV irradiation. Cells were washed twice with PBS and fixed in ice-cold 70\% ethanol overnight. After treatment with RNase (suspended in PBS, containing $0.1 \%$ Triton $\mathrm{X}-100$ and $1 \% \mathrm{BSA}$ ) at room temperature for $20 \mathrm{~min}$, cells were incubated with propidium iodide for $30 \mathrm{~min}$ in the dark followed by termination with PBS. Cell cycle distribution was analyzed by a FACSCalibur flow cytometer (BD Biosciences).

Co-IP and western blotting. HEK293T cells transfected with Flag-Poln or GFP-Poln were harvested and lysed with HEPES buffer (50 mM HEPES pH 7.5, $150 \mathrm{mM} \mathrm{NaCl}$, $1 \mathrm{mM}$ EDTA, $1 \mathrm{mM}$ EGTA, 10\% glycerol, $1 \%$ Triton X-100, $25 \mathrm{mM} \mathrm{NaF}, 10 \mu \mathrm{M}$ $\mathrm{ZnCl}_{2}$ ). The whole-cell lysates were immunoprecipitated with either anti-Flag M2 agarose or GFP-Trap A beads (Chromotek) ${ }^{6}$. Samples were separated by SDS-PAGE and detected by immunoblotting with the indicated antibodies. To confirm Poln OGlcNAcylation, 293T cells expressing Flag-Pol $\eta$ were lysed with 1xSDS lysis buffer (50 $\mathrm{mM}$ Tris-HCl pH 6.8, $100 \mathrm{mM}$ DTT, $2 \%$ SDS, $10 \%$ glycerol) at $95^{\circ} \mathrm{C}$ for $15 \mathrm{~min}$. The denaturated lysates were centrifuged and the supernatant was diluted with HEPES buffer (1:14) followed by IP with anti-Flag M2 beads. The immunoprecipitated products were separated by SDS-PAGE and analyzed with anti-O-GlcNAc antibody. To confirm that protein association happens on chromatin, HEK293T cells transfected with the indicated plasmids were harvested and permeabilized by CSK-100 buffer (100 $\mathrm{mM} \mathrm{NaCl}, 300 \mathrm{mM}$ Sucrose, $3 \mathrm{mM} \mathrm{MgCl} 2,10 \mathrm{mM}$ PIPES pH 6.8, 0.2\% Triton X-100) at $4{ }^{\circ} \mathrm{C}$ for $15 \mathrm{~min}$. The lysates were centrifuged and the supernatant was collected as the soluble fraction. The pellet was then lysed with buffer $(50 \mathrm{mM}$ HEPES, $50 \mathrm{mM}$ $\mathrm{NaCl}, 10 \%$ glycerol, $10 \mathrm{uM} \mathrm{ZnCl}, 2 \mathrm{mM} \mathrm{MgCl}_{2}, 0.05 \% \mathrm{SDS}, 0.1 \%$ Triton X-100) at $4^{\circ}$ $\mathrm{C}$ overnight before centrifugation. The supernatant was diluted with buffer $(50 \mathrm{mM}$ HEPES, $50 \mathrm{mM} \mathrm{NaCl}, 10 \%$ glycerol, $10 \mathrm{uM} \mathrm{ZnCl}, 0.1 \%$ Triton X-100) (1:10) followed by IP with anti-Flag M2 beads. For denatured chromatin fraction IP, the pellet was lysed with 1xSDS lysis buffer as above. The immunoprecipitated products were separated by SDS-PAGE and analyzed with the indicated antibodies. The relative $\mathrm{O}$ GlcNAcylation or polyubiquitination levels of Poln in each sample were represented, with the O-GlcNAcylation or polyubiquitination level of the control sample set to 1 (100\%). The gray densities of the O-GlcNAcylation or polyubiquitination signals and those of unmodified Poln were determined by the Photoshop software (Adobe Systems Incorporated, USA).

In vivo ubiquitination assay. HEK293T cells were transfected with the indicated OGT, DDB1, CDT2, or p97 siRNAs twice, and $48 \mathrm{~h}$ after the first transfection, cells were further transfected with HA-Ub and a series of Flag- or GFP-tagged Poln plasmids. The cell lysates were immunoprecipitated with Flag M2 agarose or GFPTrap A beads followed by western blotting with anti-Ubiquitin and anti-HA antibodies to detect the ubiquitination of Poln.

In vitro O-GIcNAcylation assay. pET-28a-WT or T457A Poln (with kanamycin resistance) was co-transformed with pGEX-4T-2-OGT (with ampicillin resistance) into E. coli Transetta (DE3) cells. Single clones selected on ampicillin/kanamycin plate were grown at $37^{\circ} \mathrm{C}$ in Luria-Bertani (LB) medium until they reached $\mathrm{OD}_{600}$ $=0.6$, then isopropy $\beta$-D-1-thiogalactopyranoside (IPTG) $(0.4 \mathrm{mM})$ was added and cultured at $16^{\circ} \mathrm{C}$ overnight. His-tagged Poln was affinity-purified using Ni-NTA Agarose (Qiagen) and resolved by SDS-PAGE followed by immunoblotting with antibodies against $\mathrm{O}-\mathrm{GlcNAc}$ and $\mathrm{His}^{67}$.

In vitro ubiquitination assay. Briefly, Flag-CDT2, Myc-Cul4A, Myc-DDB1, and Myc-RBX1 plasmids were co-transfected into HEK293T cells. CRL4 ${ }^{\mathrm{CDT} 2}$ complexes immobilized on Flag M2 agarose beads were washed with HEPES buffer followed by elution with Flag peptide. For the in vitro ubiquitination assay, purified WT or T457A His-Poln proteins were incubated with the eluted CRL4 CD12 complex in a ubiquitin ligation reaction mixture (final volume, $10 \mu \mathrm{l}$ ) containing $50 \mathrm{mM}$ Tris- $\mathrm{HCl}$ ( $\mathrm{pH} 7.5$ ), $5 \mathrm{mM} \mathrm{MgCl} 2,10 \mathrm{nM}$ okadaic acid, $2 \mathrm{mM}$ ATP, $0.5 \mathrm{mM}$ DTT, 5 ug ubiquitin (Boston Biochem), 60 ng E1-UbE1 (Boston Biochem), and $300 \mathrm{ng} \mathrm{E2}-\mathrm{UbcH} 5 \mathrm{c}$ (Boston Biochem) at $30^{\circ} \mathrm{C}$ for $2 \mathrm{~h}$. The reaction was terminated by boiling for $5 \mathrm{~min}$ in a SDS sample buffer, and the proteins were resolved by SDSPAGE, followed by immunoblotting with the indicated antibodies ${ }^{68}$.

Quantitative MS of Poln ubiquitination. HEK293T cells expressing WT or T457A 2xFlag-Pol $\eta$ and HA-Ub were harvested $48 \mathrm{~h}$ after plasmids' transfection and lysed with HEPES buffer (50 mM HEPES pH 7.5, $150 \mathrm{mM} \mathrm{NaCl}, 1 \mathrm{mM}$ EDTA, $1 \mathrm{mM}$ EGTA, $10 \%$ glycerol, $1 \%$ Triton X-100, $25 \mathrm{mM} \mathrm{NaF}, 10 \mu \mathrm{M} \mathrm{ZnCl}_{2}$ ). WT or T457A Poln immunoprecipitated using anti-Flag M2 agarose were eluted by $3 \times$ Flag peptide and reduced with $5 \mathrm{mM}$ DTT at $80^{\circ} \mathrm{C}$ for $10 \mathrm{~min}$ and alkylated with $10 \mathrm{mM}$ iodoacetamide in dark at room temperature for $30 \mathrm{~min}$. Proteins were resolved by SDS-PAGE (10\%) and stained with Coomassie Blue G250. Then the gel lanes were cut into two parts and digested in-gel with $12.5 \mathrm{ng} \mu l^{-1}$ of trypsin at $37^{\circ} \mathrm{C}$ for $14 \mathrm{~h}$. After extraction from the gel bands with $50 \%$ acetonitrile $(\mathrm{AcN})$ and $5 \%$ formic acid, the peptide mixtures were dried and dissolved with sample loading buffer ( $1 \%$ formic acid, $1 \%$ acetonitrile) for MS analysis.The peptide mixtures were separated and analyzed by ultra performance LC (nano Acquity Ultra Performance LC, Waters, Milford, MA) and tandem MS/MS (LTQ Orbitrap Velos, Thermo Fisher Scientific, Waltham, MA). The samples were loaded onto the column and eluted with a $1 \mathrm{~h}$ gradient with $1-35 \%$ of buffer B (buffer A: $2 \% \mathrm{AcN}$ and $0.1 \%$ formic acid; buffer B: $100 \%$ AcN $0.1 \%$ formic acid; flow rate, $\sim 300 \mathrm{nl} \mathrm{min}^{-1}$ ). Survey scans were performed in the Orbitrap analyzer at a resolution of 30,000 at $\mathrm{m} / \mathrm{z} 400$. For low molecular weight part, the 20 most intense peptide ions with charge state $\geq 2$ were subjected to fragmentation via collision-induced dissociation in the LTQ (2000 AGC target, $50 \mathrm{~ms}$ maximum ion time). In order to increase sensitivity of K462 GG-peptide, selective reaction monitoring strategy was applied as previously described $^{69}$. Targeted ions $(\mathrm{m} / \mathrm{z}=711.01, \mathrm{~m} / z=701.01)$ and the three most intense peptide ions were detected in MS/MS analysis.

The raw data files were searched by the Maxquant (version 1.5.6.5) against the Swiss-Prot reviewed database (version released in 2016.12 for human). Searching parameters consisted of full tryptic restriction, fixed modification of Cys $(+57.0215$ $\mathrm{Da})$, and variable modifications of oxidized Met $(+15.9949 \mathrm{Da})$ and di-Gly-lysine (114.0429Da). The mass tolerance was set to $20 \mathrm{ppm}$ for precursor ions and $0.5 \mathrm{Da}$ for product ions. The peptide intensity for quantification was manually analyzed by extracted ion chromatogram.

DNA fiber assay. Cells were treated with CDDP $\left(2 \mu \mathrm{g} \mathrm{ml} l^{-1}\right)$ for $24 \mathrm{~h}$. Before harvesting, cells were pulse-labeled with the modified thymidine analogues IdU $(100 \mu \mathrm{M})$ for $15 \mathrm{~min}$, followed by CIdU $(100 \mu \mathrm{M})$ for $60 \mathrm{~min}$. Cells were collected, lysed, and spread on microscope slides to obtain single-molecule DNA fibers. The labeled replication tracts were detected with primary antibodies against IdU (BD Biosciences, anti-BrdU clone B44) and CIdU (Abcam, anti-BrdU BU1/75(ICR1)) and secondary antibodies (Invitrogen, Alexa Fluor 488 goat anti-rat and Alexa Fluor 568 goat anti-mouse). Fibers were imaged using a Leica DM5000B microscope. Length of 10-150 DNA fibers were measured using the ImageJ software from $2-3$ independent experiments. $p$-Values were obtained from the Mann-Whitney test ${ }^{66}$. 
supF shuttle vector-based mutagenesis assay. Mutation frequencies were measured using the supF shuttle vector system ${ }^{49}$, which measures TLS activity in mammalian cells ${ }^{46}$. 293T cells transfected with the indicated siRNA oligos or XP30RO-lenti-GFP-Poln stable cells were transfected with a UVC-irradiated (400 J $\mathrm{m}^{-2}$ ) pSP189 reporter plasmid. Forty eight hours later, the pSP189 plasmid was retrieved from the cells using a DNA Miniprep Kit (Tiangen, China). The purified plasmid was digested with DpnI and transformed into the MBM7070 bacterial strain. The transformed MBM7070 cells were grown on LB plates containing 200 $\mu \mathrm{M}$ IPTG, $100 \mu \mathrm{g} \mathrm{ml}^{-1} \mathrm{X}$-gal, and $100 \mu \mathrm{g} \mathrm{ml}^{-1}$ ampicillin. The mutation frequency in the supF-coding region was determined by enumerating the ratios of blue (WT) and white (mutant) colonies. The pSP189 plasmid and MBM7070 strain were gifts from Dr. M. Seidman.

Cell survival assay. Cells were seeded into $6-\mathrm{cm}$ dishes ( 200 cells/dish) in triplicate and allowed to adhere for $5 \mathrm{~h}$. The cells were then treated with the indicated doses of CDDP for $24 \mathrm{~h}$ at $37^{\circ} \mathrm{C}$ or UVC irradiation. After treatment, cells were further incubated in complete medium for 7-10 days. For UVC irradiation, the cells were cultured along with caffeine $(0.4 \mathrm{mM})$ for $30 \mathrm{~min}$ prior to treatment and then incubated in complete medium supplemented with caffeine $(0.4 \mathrm{mM})$ for 7-10 days. Colonies were fixed and counted. The survival of genotoxin-exposed cells was determined by relating the cloning efficiency to that of an untreated control $^{70}$

Statistical analysis. All statistical tests were determined with a two-sided Student's $t$-test using the PRISM software (Graphpad Software Inc.) unless otherwise noted. $p$-Values were rounded to four decimal places and differences were considered as statistically significant when $p<0.05$.

Data availability. All data supporting the findings of this study are available from the corresponding authors on request.

Received: 24 April 2017 Accepted: 10 November 2017

Published online: 05 December 2017

\section{References}

1. Johnson, R. E., Prakash, S. \& Prakash, L. Efficient bypass of a thymine-thymine dimer by yeast DNA polymerase, Poleta. Science 283, 1001-1004 (1999).

2. Friedberg, E. C. et al. DNA Repair and Mutagenesis, 2nd edn. (American Society for Microbiology, Washington, DC, 2005).

3. Masutani, C. et al. The XPV (xeroderma pigmentosum variant) gene encodes human DNA polymerase eta. Nature 399, 700-704 (1999).

4. Yang, W. An overview of Y-Family DNA polymerases and a case study of human DNA polymerase eta. Biochemistry 53, 2793-2803 (2014).

5. Kannouche, P. et al. Domain structure, localization, and function of DNA polymerase eta, defective in xeroderma pigmentosum variant cells. Genes Dev. 15, 158-172 (2001).

6. Yang, W. \& Woodgate, R. What a difference a decade makes: insights into translesion DNA synthesis. Proc. Natl. Acad. Sci. USA 104, 15591-15598 (2007).

7. Bienko, M. et al. Ubiquitin-binding domains in Y-family polymerases regulate translesion synthesis. Science 310, 1821-1824 (2005).

8. Bienko, M. et al. Regulation of translesion synthesis DNA polymerase eta by monoubiquitination. Mol. Cell 37, 396-407 (2010).

9. Kannouche, P. L., Wing, J. \& Lehmann, A. R. Interaction of human DNA polymerase eta with monoubiquitinated PCNA: a possible mechanism for the polymerase switch in response to DNA damage. Mol. Cell 14, 491-500 (2004).

10. Plosky, B. S. et al. Controlling the subcellular localization of DNA polymerases iota and eta via interactions with ubiquitin. EMBO J. 25, 2847-2855 (2006).

11. Watanabe, K. et al. Rad18 guides poleta to replication stalling sites through physical interaction and PCNA monoubiquitination. EMBO J. 23, 3886-3896 (2004).

12. Albertella, M. R., Green, C. M., Lehmann, A. R. \& O'Connor, M. J. A role for polymerase eta in the cellular tolerance to cisplatin-induced damage. Cancer Res. 65, 9799-9806 (2005).

13. Chen, Y. W. et al. Human DNA polymerase eta activity and translocation is regulated by phosphorylation. Proc. Natl. Acad. Sci. USA 105, 16578-16583 (2008).

14. Kannouche, P. L. \& Lehmann, A. R. Ubiquitination of PCNA and the polymerase switch in human cells. Cell Cycle 3, 1011-1013 (2004).

15. Delbos, F. et al. Contribution of DNA polymerase eta to immunoglobulin gene hypermutation in the mouse. J. Exp. Med. 201, 1191-1196 (2005).

16. Lin, Q. et al. Increased susceptibility to UV-induced skin carcinogenesis in polymerase eta-deficient mice. Cancer Res. 66, 87-94 (2006).

17. Martomo, S. A. et al. Different mutation signatures in DNA polymerase etaand MSH6-deficient mice suggest separate roles in antibody diversification. Proc. Natl. Acad. Sci. USA 102, 8656-8661 (2005).

18. Vaisman, A., Masutani, C., Hanaoka, F. \& Chaney, S. G. Efficient translesion replication past oxaliplatin and cisplatin GpG adducts by human DNA polymerase eta. Biochemistry 39, 4575-4580 (2000).
19. Zhao, Y. et al. Structural basis of human DNA polymerase eta-mediated chemoresistance to cisplatin. Proc. Natl. Acad. Sci. USA 109, 7269-7274 (2012).

20. Ceppi, P. et al. Polymerase eta mRNA expression predicts survival of non-small cell lung cancer patients treated with platinum-based chemotherapy. Clin. Cancer Res. 15, 1039-1045 (2009).

21. Srivastava, A. K. et al. Enhanced expression of DNA polymerase eta contributes to cisplatin resistance of ovarian cancer stem cells. Proc. Natl. Acad. Sci. USA 112, 4411-4416 (2015)

22. Sale, J. E., Lehmann, A. R. \& Woodgate, R. Y-family DNA polymerases and their role in tolerance of cellular DNA damage. Nat. Rev. Mol. Cell Biol. 13, 141-152 (2012).

23. Guo, C., Kosarek-Stancel, J. N., Tang, T. S. \& Friedberg, E. C. Y-family DNA polymerases in mammalian cells. Cell Mol. Life Sci. 66, 2363-2381 (2009).

24. Ghosal, G. \& Chen, J. DNA damage tolerance: a double-edged sword guarding the genome. Transl. Cancer Res. 2, 107-129 (2013).

25. Gohler, T., Sabbioneda, S., Green, C. M. \& Lehmann, A. R. ATR-mediated phosphorylation of DNA polymerase eta is needed for efficient recovery from UV damage. J. Cell Biol. 192, 219-227 (2011).

26. Jung, Y. S., Qian, Y. \& Chen, X. DNA polymerase eta is targeted by Mdm2 for polyubiquitination and proteasomal degradation in response to ultraviolet irradiation. DNA Repair 11, 177-184 (2012).

27. Jung, Y. S., Hakem, A., Hakem, R. \& Chen, X. Pirh2 E3 ubiquitin ligase monoubiquitinates DNA polymerase eta to suppress translesion DNA synthesis. Mol. Cell Biol. 31, 3997-4006 (2011).

28. Biertumpfel, C. et al. Structure and mechanism of human DNA polymerase eta. Nature 465, 1044-1048 (2010).

29. Huang, T. T. et al. Regulation of monoubiquitinated PCNA by DUB autocleavage. Nat. Cell Biol. 8, 339-347 (2006).

30. Park, J. M. et al. Modification of PCNA by ISG15 plays a crucial role in termination of error-prone translesion DNA synthesis. Mol. Cell 54, 626-638 (2014).

31. Niimi, A. et al. Regulation of proliferating cell nuclear antigen ubiquitination in mammalian cells. Proc. Natl. Acad. Sci. USA 105, 16125-16130 (2008)

32. Hart, G. W., Slawson, C., Ramirez-Correa, G. \& Lagerlof, O. Cross talk between O-GlcNAcylation and phosphorylation: roles in signaling, transcription, and chronic disease. Annu. Rev. Biochem. 80, 825-858 (2011).

33. Bond, M. R. \& Hanover, J. A. A little sugar goes a long way: the cell biology of O-GlcNAc. J. Cell Biol. 208, 869-880 (2015).

34. Chen, Q., Chen, Y., Bian, C., Fujiki, R. \& Yu, X. TET2 promotes histone O-GlcNAcylation during gene transcription. Nature 493, 561-564 (2013).

35. Xu, Q. et al. AMPK regulates histone H2B O-GlcNAcylation. Nucleic Acids Res. 42, 5594-5604 (2014).

36. de Queiroz, R. M., Carvalho, E. \& Dias, W. B. O-GlcNAcylation: the sweet side of the cancer. Front. Oncol. 4, 132 (2014).

37. Slawson, C. \& Hart, G. W. O-GlcNAc signalling: implications for cancer cell biology. Nat. Rev. Cancer 11, 678-684 (2011).

38. Chen, Q. \& Yu, X. OGT restrains the expansion of DNA damage signaling. Nucleic Acids Res. 44, 9266-9278 (2016).

39. Wang, P. et al. OGT mediated histone H2B S1 12 GlcNAcylation regulates DNA damage response. J. Genet. Genomics 42, 467-475 (2015).

40. Zachara, N. E., Molina, H., Wong, K. Y., Pandey, A. \& Hart, G. W. The dynamic stress-induced "O-GlcNAc-ome" highlights functions for O-GlcNAc in regulating DNA damage/repair and other cellular pathways. Amino Acids $\mathbf{4 0}$ 793-808 (2011)

41. Lv, L. et al. Mismatch repair protein MSH2 regulates translesion DNA synthesis following exposure of cells to UV radiation. Nucleic Acids Res. 41, 10312-10322 (2013).

42. Han, J. et al. SIVA1 directs the E3 ubiquitin ligase RAD18 for PCNA monoubiquitination. J. Cell Biol. 205, 811-827 (2014).

43. Ramakrishnan, P. et al. Activation of the transcriptional function of the NFkappaB protein c-Rel by O-GlcNAc glycosylation. Sci. Signal. 6, ra75 (2013).

44. Diamant, N. et al. DNA damage bypass operates in the S and G2 phases of the cell cycle and exhibits differential mutagenicity. Nucleic Acids Res. 40, 170-180 (2012).

45. Davis, E. J. et al. DVC1 (C1orf124) recruits the $\mathrm{p} 97$ protein segregase to sites of DNA damage. Nat. Struct. Mol. Biol. 19, 1093-1100 (2012).

46. Mosbech, A. et al. DVC1 (C1orf124) is a DNA damage-targeting p97 adaptor that promotes ubiquitin-dependent responses to replication blocks. Nat. Struct. Mol. Biol. 19, 1084-1092 (2012).

47. Franz, A., Ackermann, L. \& Hoppe, T. Ring of change: CDC48/p97 drives protein dynamics at chromatin. Front. Genet. 7, 73 (2016).

48. Matsuda, T., Bebenek, K., Masutani, C., Hanaoka, F. \& Kunkel, T. A. Low fidelity DNA synthesis by human DNA polymerase-eta. Nature 404, 1011-1013 (2000).

49. Parris, C. N. \& Seidman, M. M. A signature element distinguishes sibling and independent mutations in a shuttle vector plasmid. Gene 117, 1-5 (1992).

50. Kim, S. H. \& Michael, W. M. Regulated proteolysis of DNA polymerase eta during the DNA-damage response in C. elegans. Mol. Cell 32, 757-766 (2008). 
51. Tsanov, N. et al. PIP degron proteins, substrates of CRL4Cdt2, and not PIP boxes, interfere with DNA polymerase eta and kappa focus formation on UV damage. Nucleic Acids Res. 42, 3692-3706 (2014).

52. Kim, W. et al. Systematic and quantitative assessment of the ubiquitin-modified proteome. Mol. Cell 44, 325-340 (2011).

53. Shachar, S. et al. Two-polymerase mechanisms dictate error-free and errorprone translesion DNA synthesis in mammals. EMBO J. 28, 383-393 (2009).

54. Dantuma, N. P., Acs, K. \& Luijsterburg, M. S. Should I stay or should I go: $\mathrm{VCP} / \mathrm{p} 97-$ mediated chromatin extraction in the DNA damage response. Exp. Cell Res. 329, 9-17 (2014).

55. Dai, X., You, C. \& Wang, Y. The functions of serine 687 phosphorylation of human DNA polymerase eta in UV damage tolerance. Mol. Cell. Proteomics 15, 1913-1920 (2016).

56. Despras, E. et al. Rad18-dependent SUMOylation of human specialized DNA polymerase eta is required to prevent under-replicated DNA. Nat. Commun. 7, 13326 (2016).

57. Ferrer, C. M., Sodi, V. L. \& Reginato, M. J. O-GlcNAcylation in cancer biology: Linking metabolism and signaling. J. Mol. Biol. 428, 3282-3294 (2016).

58. Kawamoto, T. et al. Dual roles for DNA polymerase eta in homologous DNA recombination and translesion DNA synthesis. Mol. Cell 20, 793-799 (2005).

59. Lehmann, A. R. New functions for Y family polymerases. Mol. Cell 24, 493-495 (2006).

60. Garcia-Exposito, L. et al. Proteomic profiling reveals a specific role for translesion DNA polymerase eta in the alternative lengthening of telomeres. Cell Rep. 17, 1858-1871 (2016).

61. McIlwraith, M. J. et al. Human DNA polymerase eta promotes DNA synthesis from strand invasion intermediates of homologous recombination. Mol. Cell 20, 783-792 (2005)

62. Liu, Y. et al. Variants of mouse DNA polymerase kappa reveal a mechanism of efficient and accurate translesion synthesis past a benzo[a]pyrene dG adduct. Proc. Natl. Acad. Sci. USA 111, 1789-1794 (2014).

63. Xu, T. et al. ProLuCID: $\mathrm{n}$ improved SEQUEST-like algorithm with enhanced sensitivity and specificity. J. Proteomics 129, 16-24 (2015).

64. Tabb, D. L., McDonald, W. H. \& Yates, J. R. 3rd DTASelect and Contrast: tools for assembling and comparing protein identifications from shotgun proteomics. J. Proteome Res. 1, 21-26 (2002).

65. Yang, B. et al. Identification of cross-linked peptides from complex samples. Nat. Methods 9, 904-906 (2012).

66. Yang, Y. et al. FANCD2 and REV1 cooperate in the protection of nascent DNA strands in response to replication stress. Nucleic Acids Res. 43, 8325-8339 (2015).

67. Guo, B. et al. O-GlcNAc-modification of SNAP-29 regulates autophagosome maturation. Nat. Cell Biol. 16, 1215-1226 (2014).

68. Furukawa, M., Andrews, P. S. \& Xiong, Y. Assays for RING family ubiquitin ligases. Methods Mol. Biol. 301, 37-46 (2005).

69. Xu, P. et al. Quantitative proteomics reveals the function of unconventional ubiquitin chains in proteasomal degradation. Cell 137, 133-145 (2009).

70. Zhang, X. et al. Mouse DNA polymerase kappa has a functional role in the repair of DNA strand breaks. DNA Repair (Amst.) 12, 377-388 (2013).

\section{Acknowledgements}

The authors thank Drs. Alan Lehmann, Simone Sabbioneda, Jun Huang, Wei Li, Hengyu Fan, and Jun Dou for reagents; Dr. Wei Yang, Dr. Yungui Yang, and Dr. Wei Li for helpful discussion; and Miss Qing Meng for cell sorting through FACS. This work was supported by NSFC81630078, 91754204, MOST2017YFC1001001, 2013CB911201 and 2014CB84980001, NSFC31471331, 31670822, 31570816, 91519324, 81371415, 31470784, and 31701227; CAS Strategic Priority Research Program XDB14030300; the State Key Laboratory of Membrane Biology; CNU interdisciplinary project; and Beijing Nova Program Interdisciplinary Cooperation Project (Z161100004916042).

\section{Author contributions}

X.M. performed most of the experiments with help from H.L. and J.L.; Y.W. performed ubiquitination mass spectrometric analysis under the supervision of P.X.; Y.-H.D. performed O-GlcNAcylation mass spectrometric analysis under the supervision of M.-Q.D.; H.S. performed DNA fiber assay; Y.Y. performed cell survival assay; C.S., M.H., Y.L., and Y.T. constructed Pol $\eta$ mutant plasmids; Y.Z. provided discussion and proofread the manuscript. X.M., T.-S.T., and C.G. designed the whole project, analyzed the data, and wrote the manuscript with input from all authors.

\section{Additional information}

Supplementary Information accompanies this paper at https://doi.org/10.1038/s41467017-02164-1.

Competing interests: The authors declare no competing financial interests.

Reprints and permission information is available online at http://npg.nature.com/ reprintsandpermissions/

Publisher's note: Springer Nature remains neutral with regard to jurisdictional claims in published maps and institutional affiliations.

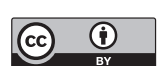

Open Access This article is licensed under a Creative Commons Attribution 4.0 International License, which permits use, sharing, adaptation, distribution and reproduction in any medium or format, as long as you give appropriate credit to the original author(s) and the source, provide a link to the Creative Commons license, and indicate if changes were made. The images or other third party material in this article are included in the article's Creative Commons license, unless indicated otherwise in a credit line to the material. If material is not included in the article's Creative Commons license and your intended use is not permitted by statutory regulation or exceeds the permitted use, you will need to obtain permission directly from the copyright holder. To view a copy of this license, visit http://creativecommons.org/ licenses/by/4.0/.

(c) The Author(s) 2017 